\title{
REVIEW
}

\section{Management of Pemphigus Vulgaris}

\author{
Mimansa Cholera $\cdot$ Nita Chainani-Wu
}

Received: April 12, 2016 / Published online: June 10, 2016

(C) The Author(s) 2016. This article is published with open access at Springerlink.com

\section{ABSTRACT}

Introduction: Pemphigus vulgaris (PV) is a chronic, autoimmune, vesiculobullous disease. As a result of the relative rarity of PV, published randomized controlled trials (RCTs) are limited, which makes it difficult to evaluate the efficacy of different treatment regimens in this disease. This also precludes conduct of a meta-analysis. Methods: English-language publications describing treatment outcomes of patients with PV were identified by searches of electronic databases through May 2015, and additionally by review of the bibliography of these publications. A total of 89 papers, which included 21 case reports, 47 case series, 8 RCTs, and 13 observational studies, were identified. The findings from these publications, including information on disease course and prognosis, medications used, treatment responses, and side

Enhanced content To view enhanced content for this article go to http://www.medengine.com/Redeem/ A1D4F0601A5546F4.

M. Cholera $\cdot$ N. Chainani-Wu ( $\square)$

Private Practice in Oral Medicine Clinic, 100 West El Camino Real Suite 76, Mountain View, CA 94040, USA

e-mail: nitacwu@gmail.com effects, are summarized in the tables and text of this review.

Results: Prior to availability of corticosteroid therapy, PV had a high fatality rate. Early publications from the 1970s reported high-dose, prolonged corticosteroid use and significant associated side effects. Later reports described use of corticosteroids along with steroid-sparing adjuvants, which allows a reduction in the total dose of corticosteroids and a reduction in observed mortality and morbidity. For the majority of patients in these reports, a long-term course on medications lasting about 5-10 years was observed; however, subgroups of patients requiring shorter courses or needing longer-term therapy have also been described. Early diagnosis of PV and early initiation of treatment were prognostic factors. In recent publications, commonly used initial regimens include corticosteroids in combination with mycophenolate or azathioprine; whereas, for patients with inadequate response to these regimens, adjuvants such as intravenous immunoglobulin (IVIg) or rituximab are used.

Conclusion: The review findings emphasize the importance of early diagnosis, early initiation of 
treatment, and use of steroid-sparing adjuvants to allow a reduced total dose and duration on corticosteroids. Also highlighted is the need for more RCTs.

Keywords: Autoimmune vesiculobullous disease; Azathioprine; Corticosteroids; Methotrexate and IVIg; Mycophenolate mofetil; Pemphigus vulgaris; Rituximab

\section{INTRODUCTION}

Pemphigus vulgaris (PV) is a chronic, autoimmune, mucocutaneous, vesiculobullous disease [1].

The word pemphigus comes from the Greek word pemphix, which means blister [2]. It is a rare disease with estimated worldwide annual incidence of 0.1-0.5 per 100,000 [3]. It occurs in all racial and ethnic groups with the highest incidence seen in Ashkenazi Jews [4]. Occurrence is most common during the fifth and sixth decades of life, although a few cases have been reported in children [5].

In the majority of cases, PV initially presents with lesions on the oral mucosa [3]. Often the first sites affected are those exposed to frictional trauma including the buccal and lateral tongue mucosa along the occlusal level, or the gingiva, but PV can occur on any oral site particularly if exposed to sharp or acidic foods. The lesions start as vesicles which rupture easily leaving erosions and ulcers.

The pathogenesis of pemphigus involves the presence of circulating and tissue-bound autoantibodies to the keratinocyte cell surface desmosomal molecules desmoglein 3 (Dsg3) and desmoglein 1 (Dsg1). Dsg3 and Dsg1 belong to the cadherin superfamily involved in cell-cell adhesion. These autoantibodies cause loss of cell-cell adhesion between epithelial cells, which results in suprabasilar intraepithelial vesicle formation $[4,6]$.

Diagnostic tests include perilesional mucosal or skin biopsy for histologic examination and direct immunofluoresence testing. Histologic findings include presence of intraepithelial blisters and suprabasilar acantholysis; direct immunofluorescence findings include IgG deposits and less commonly $\operatorname{IgM}$ and $\mathrm{C} 3$ deposits in intercellular spaces in the epithelium. Blood tests include ELISA testing for Dsg3 and Dsg1 autoantibodies [7].

Prior to availability of corticosteroid therapy in the 1950s, PV had a very high fatality rate. While many treatment options are now available, corticosteroids in combination with other drugs still form the mainstay of treatment. Mortality from pemphigus has decreased significantly in the last half century and is now usually due to adverse effects of the medications used $[8,9]$.

As a result of the relative rarity of pemphigus, there are very few randomized controlled trials. However, numerous observational studies, case reports, and case series have been published that report on the treatment of pemphigus. The objective of this review was to summarize the findings from all of the reported human studies including observational studies and case reports.

\section{METHODS}

Publications relating to treatment of PV were identified by searches of electronic databases including PubMed, Cochrane, and Google Scholar through May 2015. Keywords used included pemphigus vulgaris, autoimmune vesiculobullous disease, corticosteroids, azathioprine, rituximab, mycophenolate mofetil, methotrexate, and IVIg. The full-text 
versions of the papers identified were obtained. The bibliography of these papers was also reviewed to identify any additional papers that did not appear in the electronic search. Only English-language papers describing treatment outcomes of patients with PV were included in this review. A total of 89 papers, which included 21 case reports, 47 case series, 8 RCTs, and 13 observational studies, were included. These papers were reviewed to obtain information on publication date, type of study done, age of the patients, extent of lesion involvement (skin and mucosa), previous treatments if any, medications used, duration of use of previous medications before new ones were started, duration to first improvement after the start of medications, follow-up duration, concomitant medication used along with main drug, outcome, duration on medication, adverse effects of drugs, and antibody titer changes after treatment. This information is summarized in Tables 1, 2, 3, 4, 5 and 6.

Definitions for some of the terms relating to treatment outcomes listed in the tables are described in a consensus statement published in 2008 [10] as follows:

Complete remission off therapy: Absence of new and/or established lesions while the patient is off all systemic therapy for at least 2 months.

Complete remission on therapy: Absence of new or established lesions while the patient is receiving minimal therapy.

Minimal therapy: Less than, or equal to, $10 \mathrm{mg} /$ day of prednisone (or the equivalent) and/or minimal adjuvant therapy for at least 2 months.

Minimal adjuvant therapy: Half of the dose required to be defined as treatment failure.

Failure of therapy: Failure to control disease activity (i.e., relapse/flare) with full therapeutic doses of systemic treatments.
Partial remission off therapy: Presence of transient new lesions that heal within 1 week without treatment and while the patient is off all systemic therapy for at least 2 months.

Partial remission on minimal therapy: Presence of transient new lesions that heal within 1 week while the patient is receiving minimal therapy, including topical steroids [10].

However not all papers included in this review have described their specific definition for these terms. If these terms were mentioned in the publication, we have listed them in the tables as mentioned in the publication.

This article is based on previously conducted studies and does not involve any studies of human or animal subjects performed by any of the authors.

\section{RESULTS}

\section{Corticosteroids (CS)}

Since the time of their approval in the 1950s, corticosteroids have been the mainstay of treatment of PV.

\section{Mechanism of Action}

Corticosteroids have strong anti-inflammatory and immunosuppressive effects. They affect almost every aspect of the immune system. They are potent inhibitors of NFkappa B activation and have effects on leukocyte movement, leukocyte function, and humoral factors. In addition they have inhibitory effects on many known cytokines [11].

The first case series on corticosteroid use in PV was published in 1972.

The publications reporting use of corticosteroids in PV are summarized in Table 1. This table includes papers that had systemic corticosteroids as the primary 


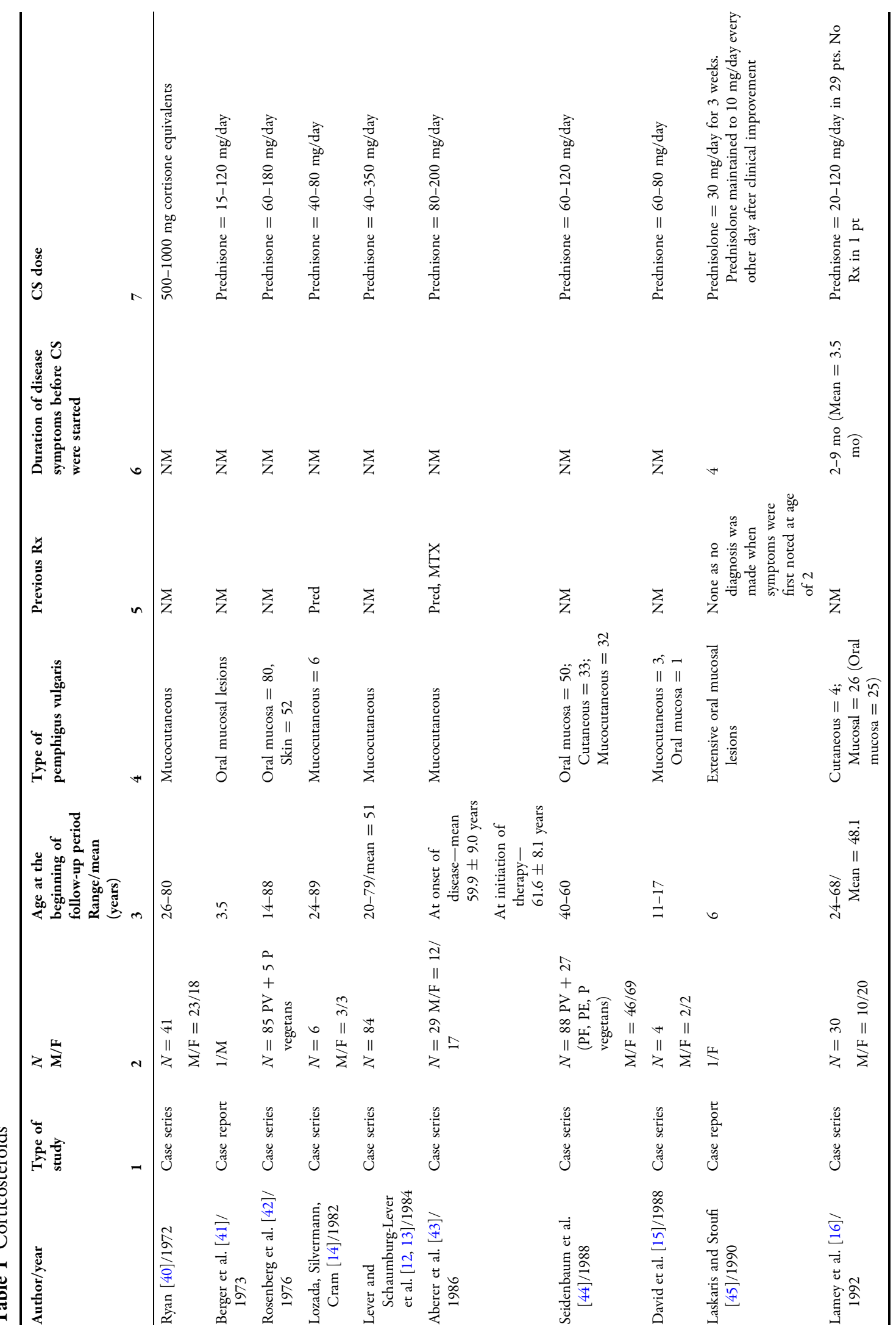




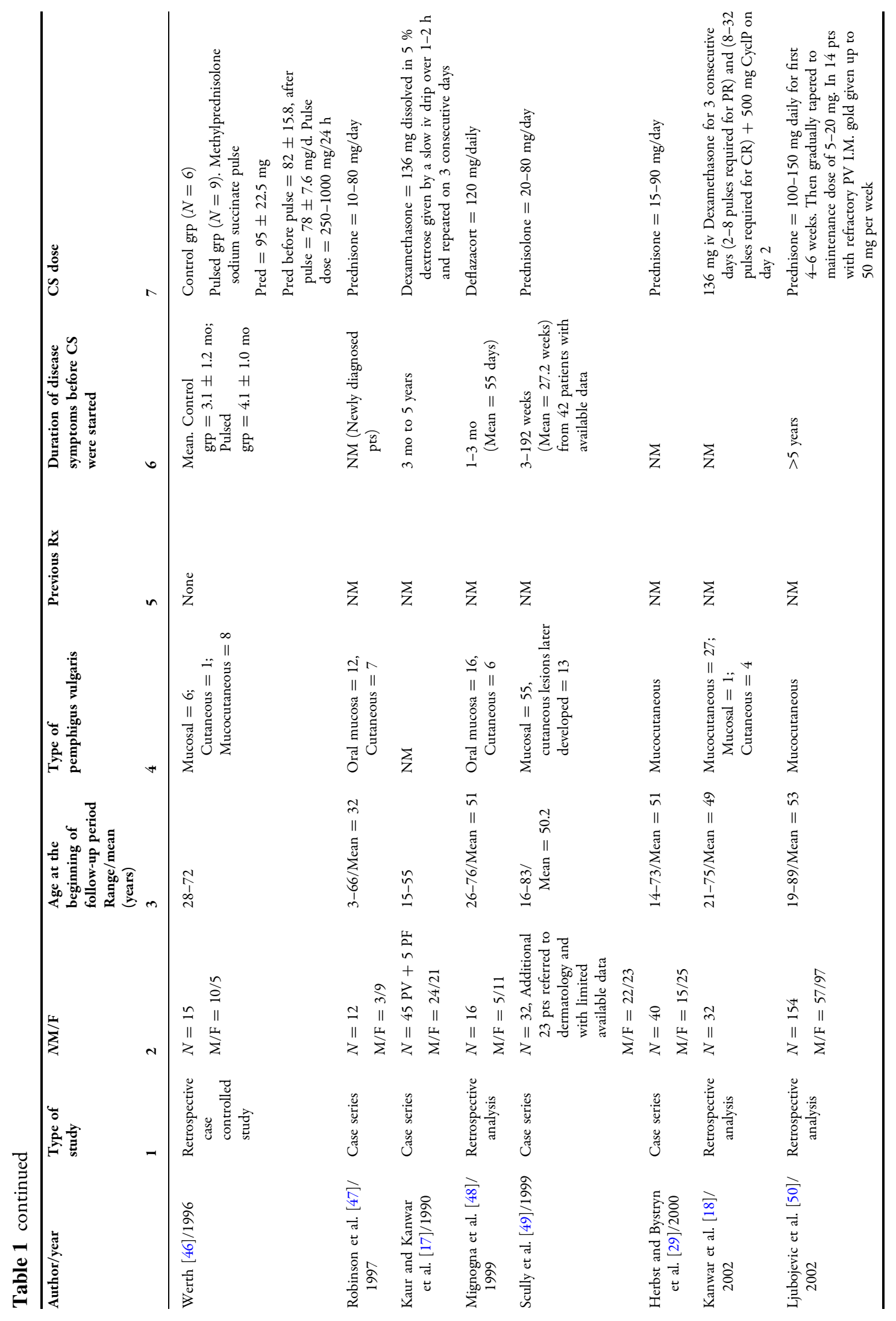




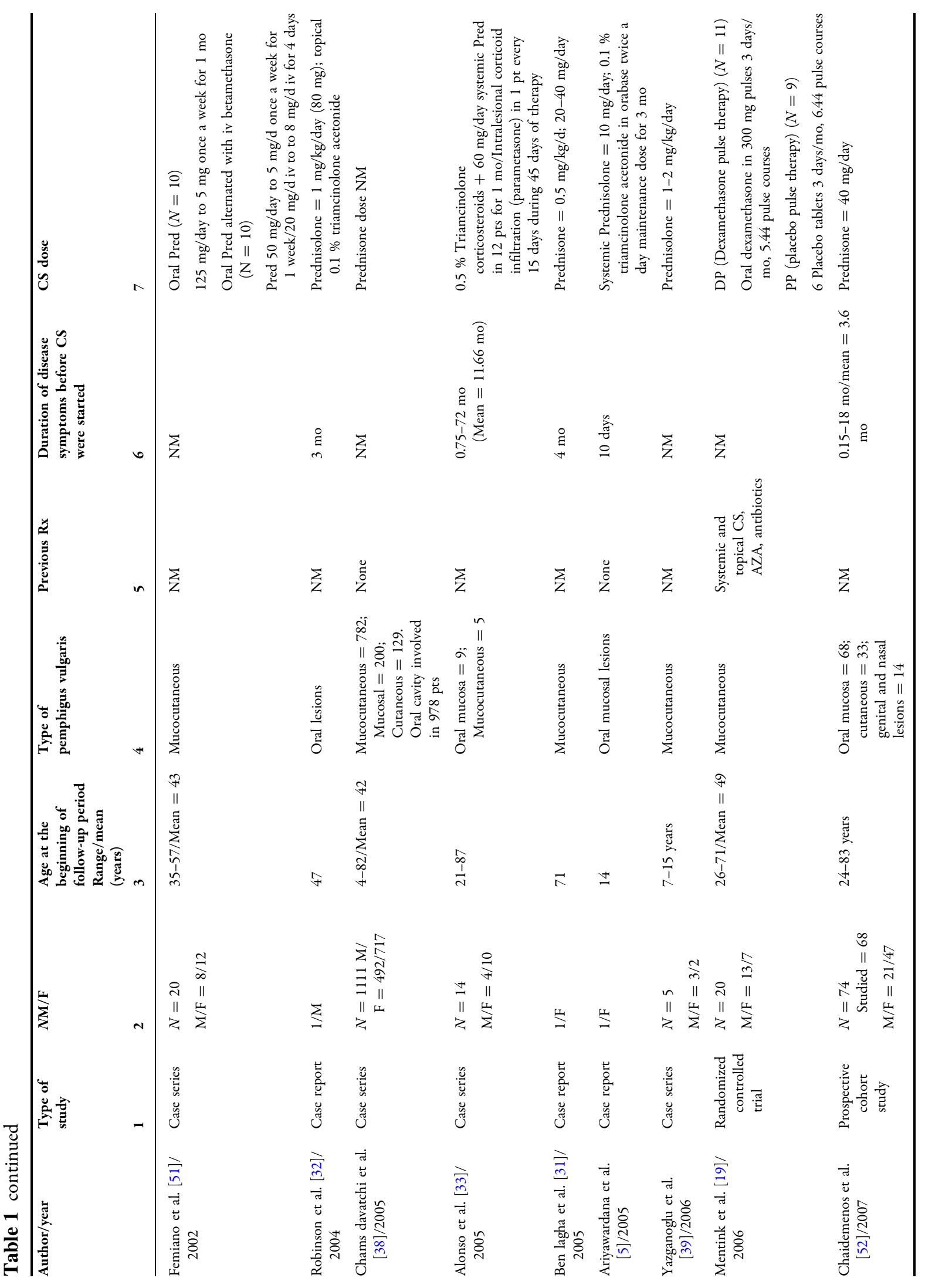




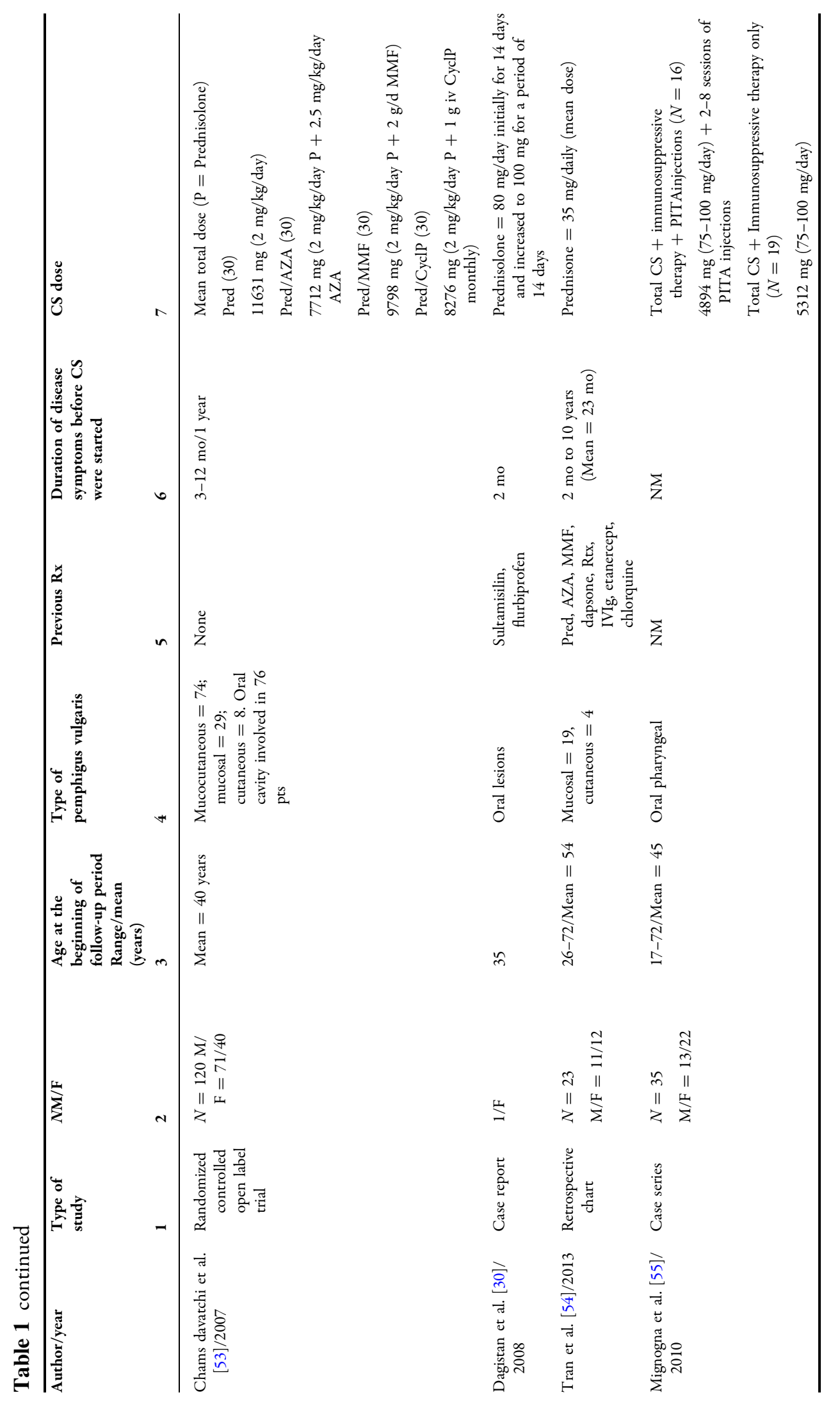




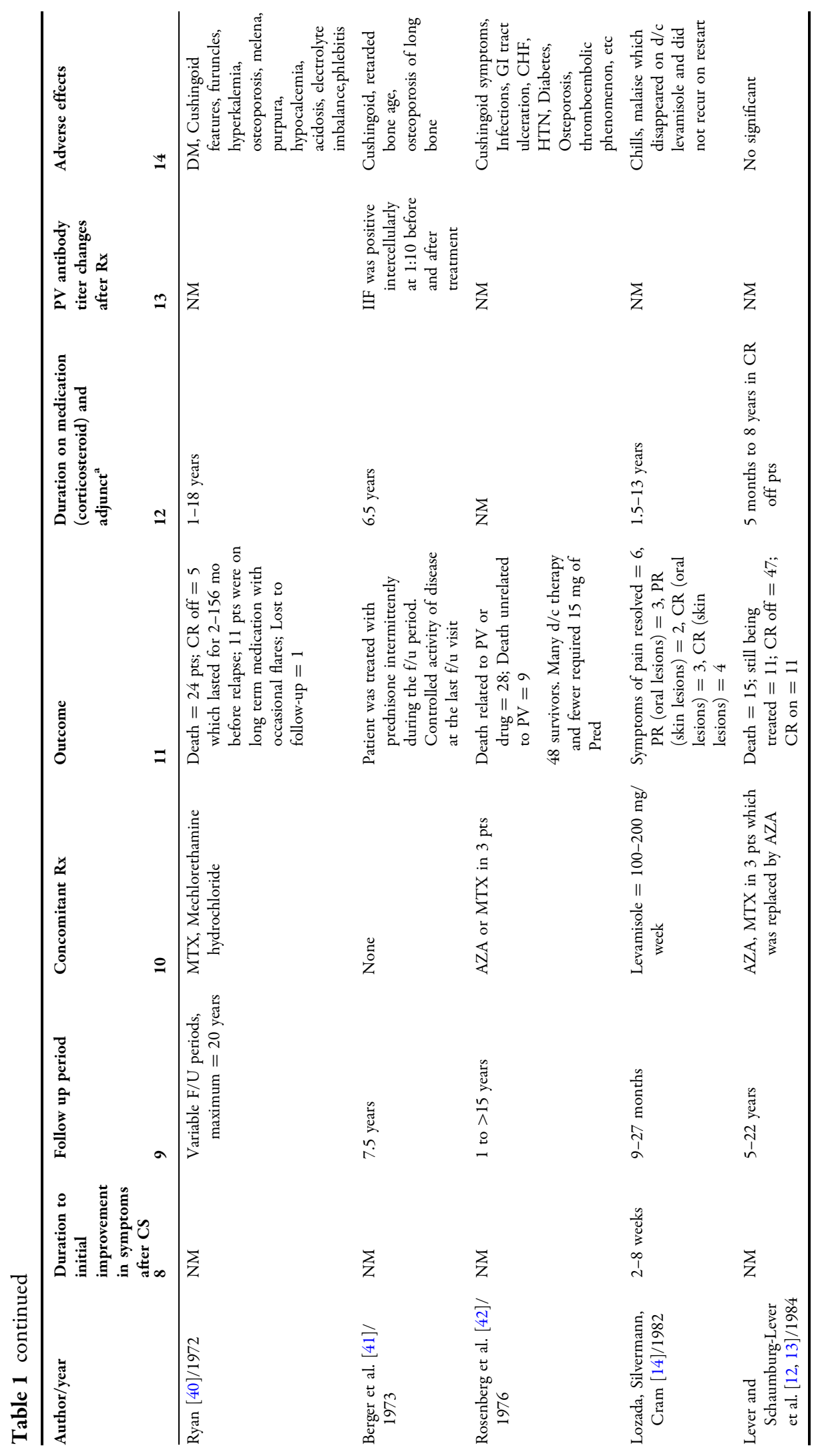




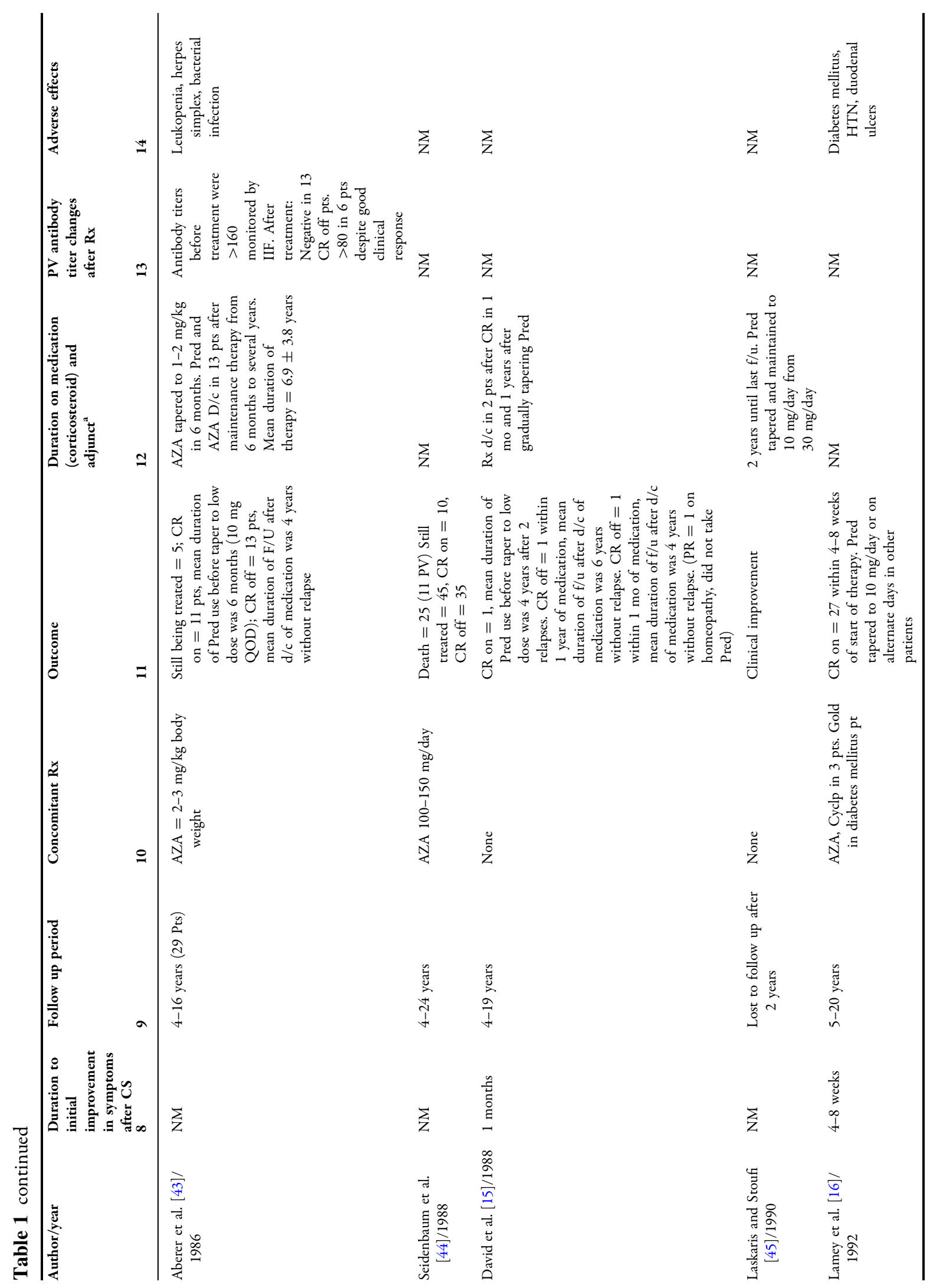




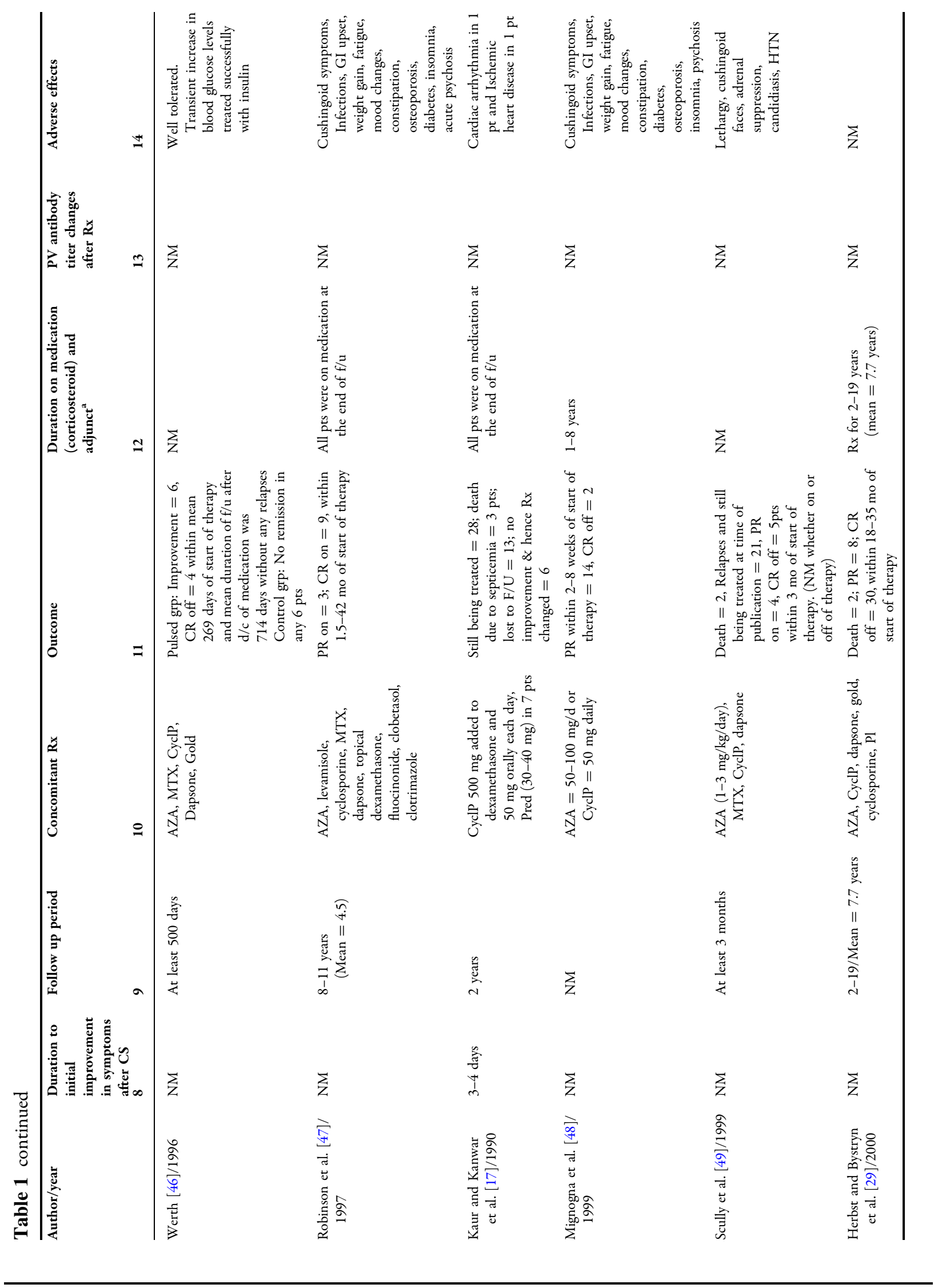




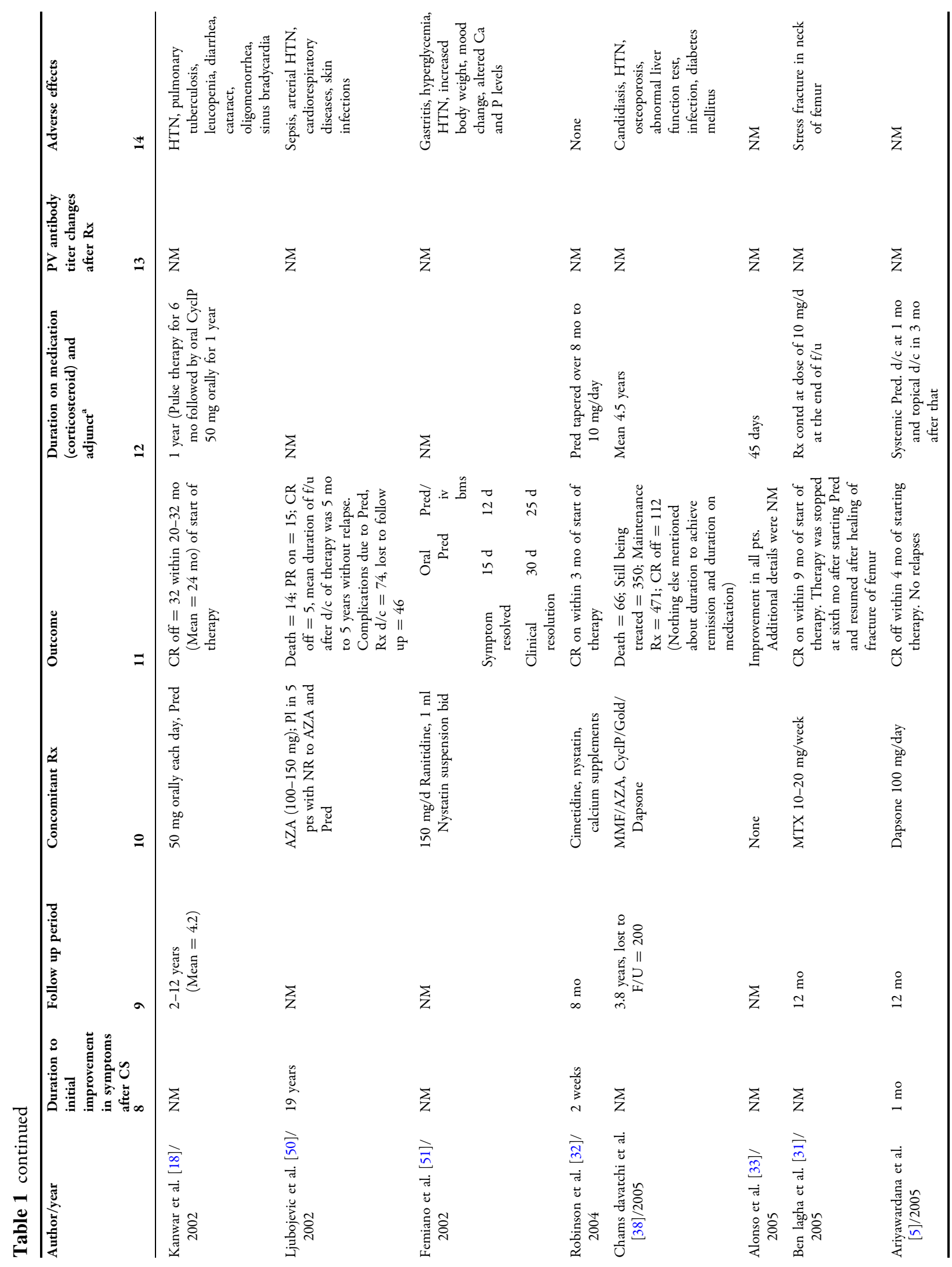




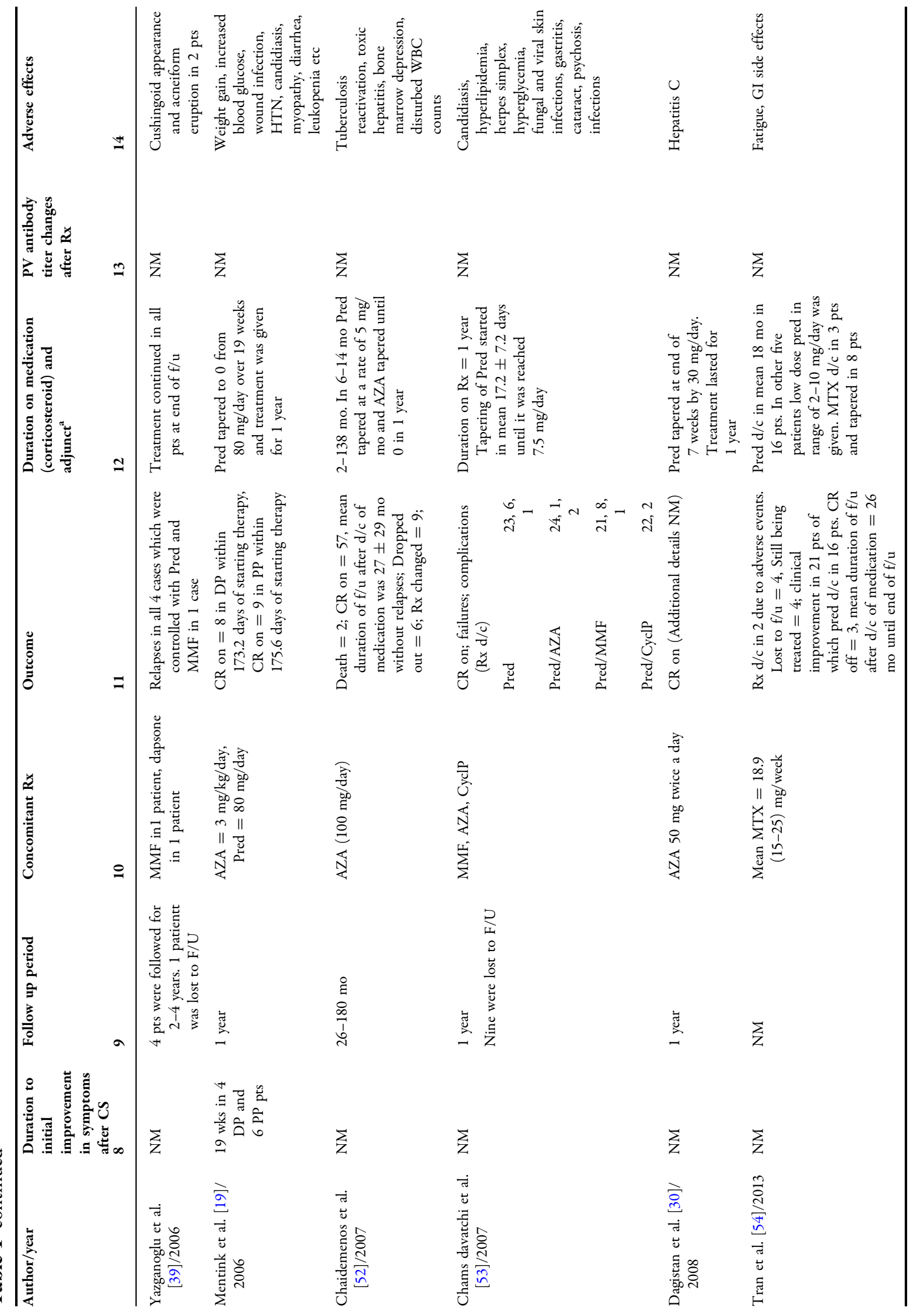




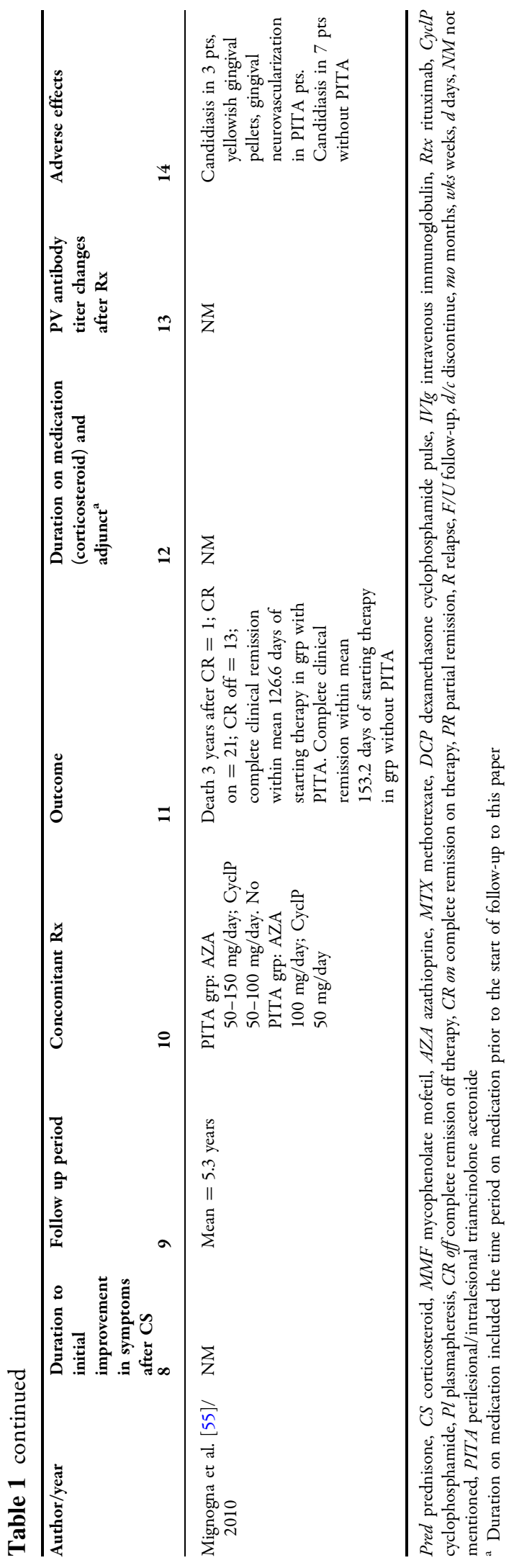

medication used. Topical steroids were also used in many of the reports. In addition, adjuvant drugs were added in most cases. These adjuvants included azathioprine, methotrexate, cyclophosphamide, dapsone, gold, levamisole, cyclosporine, and mycophenolate. Adjuvants were usually administered one at a time; however, they were changed when lack of response was noted, and therefore some patients had multiple adjuvants used sequentially over the period of treatment.

\section{Publication Type, Patient Profiles, and Sample} Sizes

Seventeen case series were found, with the number of cases included in the individual papers ranging from 4 to 1111 cases (a total of 1704 patients were included in the 17 case series, of which 1681 had PV and 23 had either pemphigus foliaceous, pemphigus vegetans, or pemphigus erythematous). Six case reports describing single patients, one prospective cohort study $(n=74)$, two randomized controlled trials $(n=20$ and $n=120)$, and five retrospective cohort studies $(n=15, n=16$, $n=23, n=32$, and $n=154)$ are summarized in the Table 1. In all, the total number of cases in these 31 publications was 2164 out of which 2141 were PV patients, and the rest had pemphigus foliaceous or pemphigus vegetans or pemphigus erythematous. These 31 reports originated from the USA, Israel, Iran, Sri Lanka, India, Scotland, Italy, Greece, Spain, the Netherlands, Germany, France, Singapore and Turkey.

Age at initial diagnosis of $\mathrm{PV}$ in these publications ranged from 4 to 89 years.

\section{Medication Use}

Prednisone and prednisolone were the most commonly used corticosteroids. Starting doses 
ranged from 15 to $180 \mathrm{mg}$ prednisone equivalent daily in all but one of the reports where doses as high as $400 \mathrm{mg}$ daily were used $[12,13]$.

\section{Duration of PV Before Corticosteroids Were Started}

This ranged from 0.15 months to 6 years.

\section{Duration of Total Follow-up}

Duration of total clinical follow-up of the individual patients ranged from 9 months to 22 years.

\section{Duration Before Any Clinical Improvement Was Noted}

Seven publications reported on the duration before any clinical improvement after the start of corticosteroids was apparent, and this ranged from 3 days to 19 weeks [14-20].

\section{Duration to Start of Taper of Corticosteroids}

Information regarding tapering of corticosteroids was reported in seven publications. The duration before the start of taper of corticosteroids ranged from 0.5 to 12 months in these seven publications comprising of 156 patients.

\section{Duration to Complete Remission (On and Off Therapy)}

Duration to complete remission on therapy was reported in 15 articles, and ranged from 1.5 to 42 months (3.5 years), in 797 patients.

Duration to complete remission off therapy was reported in 15 articles, and ranged from 4 to 120 months (10 years) in 321 patients.

\section{Remission}

Of a total of 2141 patients reported on in Table 1, at the end of follow-up 97 patients had achieved partial remission on therapy, 797 patients had achieved complete remission on therapy, and 321 patients had achieved complete remission off therapy. A total of 485 patients were still being treated at the time of publication, 156 patients were lost to follow-up, death occurred in 177 patients, and 47 patients were classified as non-responders and referred elsewhere for treatment.

\section{Duration of Medication Use}

Total duration of medication use for all reported patients including those still on therapy at the time of publication ranged from 1.5 to 240 months (20 years).

\section{Follow-up Duration After Discontinuation of Medications}

Follow-up ranged from 2 to 156 months (13 years) after discontinuation of treatment in the 321 patients with complete remission off therapy, during which time there was no recurrence.

\section{Mortality}

Death occurred in a total of 177 of 2141 patients (8.26\%) with PV in all reports. These included deaths from all causes. Of these, the reports published between 1970 and 1980 included 127 patients with 61 deaths (48.03\%), between 1981 and 1990 included 183 patients with 26 deaths (14.2\%), between 1991 and 2000 included 190 patients with 7 deaths (3.6\%), and those published between 2001 and 2010 included 1589 patients with 83 deaths (5.2\%).

\section{Adverse Effects}

Adverse effects from corticosteroids reported in these papers included Cushingoid symptoms, diabetes mellitus, osteoporosis, hypertension, insomnia, GI upset, increased weight, candidiasis, tuberculosis, mood change, abnormal liver function test, fungal and viral infection, fatigue, acute psychosis, 
hyperglycemia, electrolyte imbalance, hypocalcemia, acidosis, hyperkalemia, phlebitis, herpes simplex, hyperlipidemia, bone marrow depression, cataract, and myopathy.

\section{Azathioprine (AZA)}

Azathioprine was approved by the US Food and Drug Administration (FDA) in 1968 as an immunosuppressant to prevent organ transplant rejection.

\section{Mechanism of Action}

This drug restricts synthesis of DNA, RNA, and proteins by inhibiting metabolism of purine. It also interferes with cellular metabolism and mitosis [8].

Publication Type, Patient Profiles, and Sample Sizes

The studies reporting use of AZA in PV are summarized in Tables 1 and 2. Of the 31 papers in Table 1, 17 had included azathioprine as one of the treatment modalities. Table 2 includes only those publications that reported on comparative analyses of outcomes for patients on prednisone alone vs. those on prednisone in combination with azathioprine. The first case series on use of AZA in PV was published in 1986.

One randomized double blind controlled study $(n=56)$ and two retrospective cohort studies ( $n=48$ and $n=36)$ are summarized in Table 2. In all, a total of 140 patients were included in these three reports.

Age at initial diagnosis of PV in these publications ranged from 16 to 83 years.

\section{Medication Use}

The dosage of azathioprine used was $40 \mathrm{mg}$ /day up to $3 \mathrm{mg} / \mathrm{kg} /$ day in all reports. Prednisone was used concomitantly with azathioprine in all reports. Azathioprine was added at the onset of treatment in the three reports in Table 2 and sometime after onset of corticosteroid use in the reports in Table 1.

\section{Duration of PV Before Azathioprine Was}

Started in the Reports Summarized in Table 2

This ranged from 4 to 10 months.

\section{Duration of Follow-up in the Reports \\ Summarized in Table 2}

Duration of clinical follow-up of the individual patients on azathioprine in these reports ranged from 12 months to 10 years.

\section{Duration to Complete Remission (On and Off therapy) for the Azathioprine Plus Prednisone Group in Table 2}

Duration to complete remission on therapy was reported in three articles and, ranged from 6 to 12 months, in 67 patients.

Duration to complete remission off therapy was reported in two articles and, ranged from 6 to 12 months, in eight patients.

Patients on prednisone and azathioprine had better responses as compared to patients on prednisone alone, with more patients achieving remission, and with fewer side effects.

\section{Remission}

Of a total of 140 patients, at the end of follow-up 11 patients had achieved partial remission and mean duration to achieve that was 234.4 days, 67 patients had achieved complete remission on therapy, and eight patients had achieved complete remission off therapy. Six patients were still being treated at the time of publication. No response was seen in 17 patients. Treatment failed in five patients. Death occurred in 13 patients and 13 patients were lost to follow-up. 


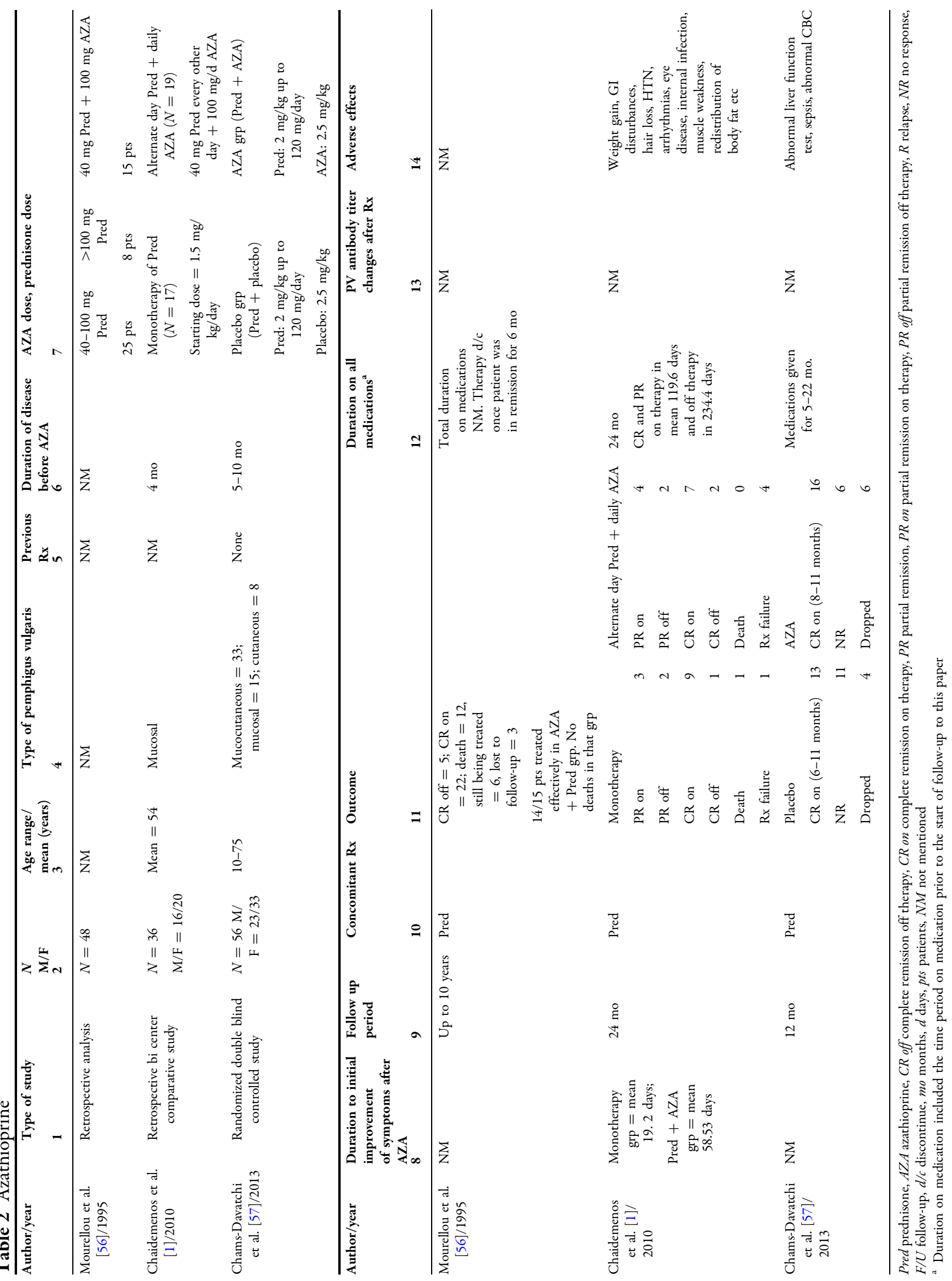




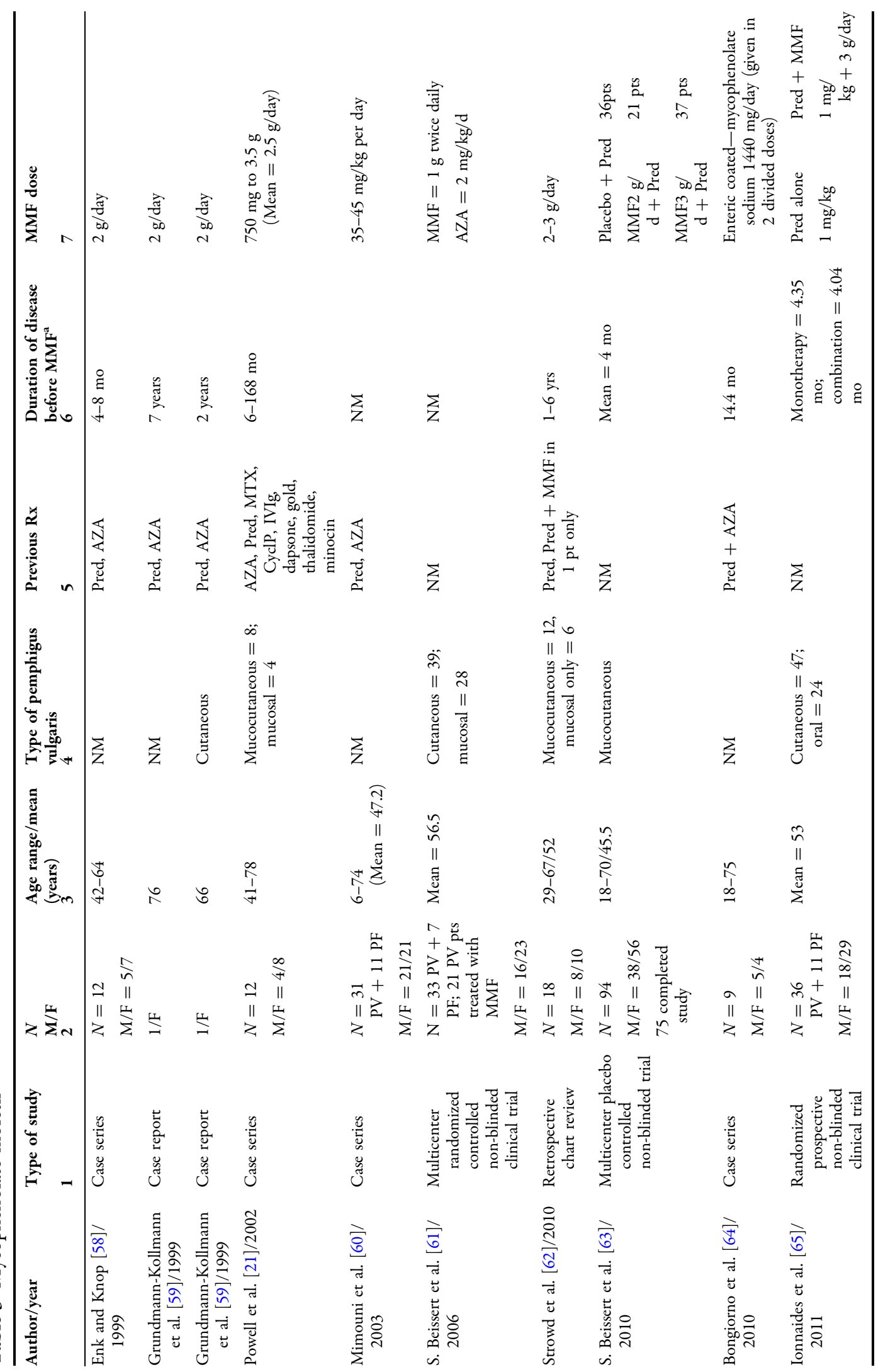




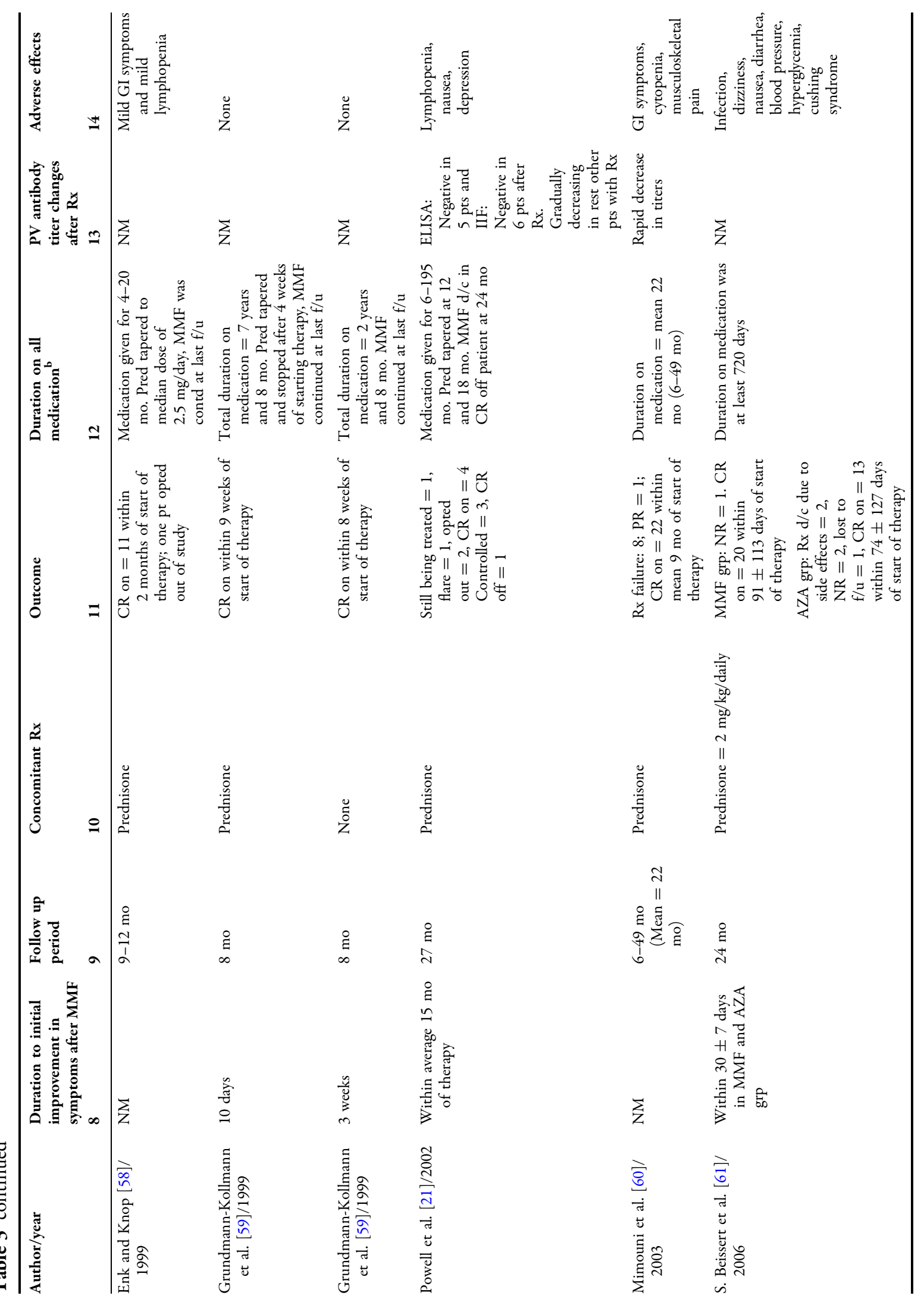




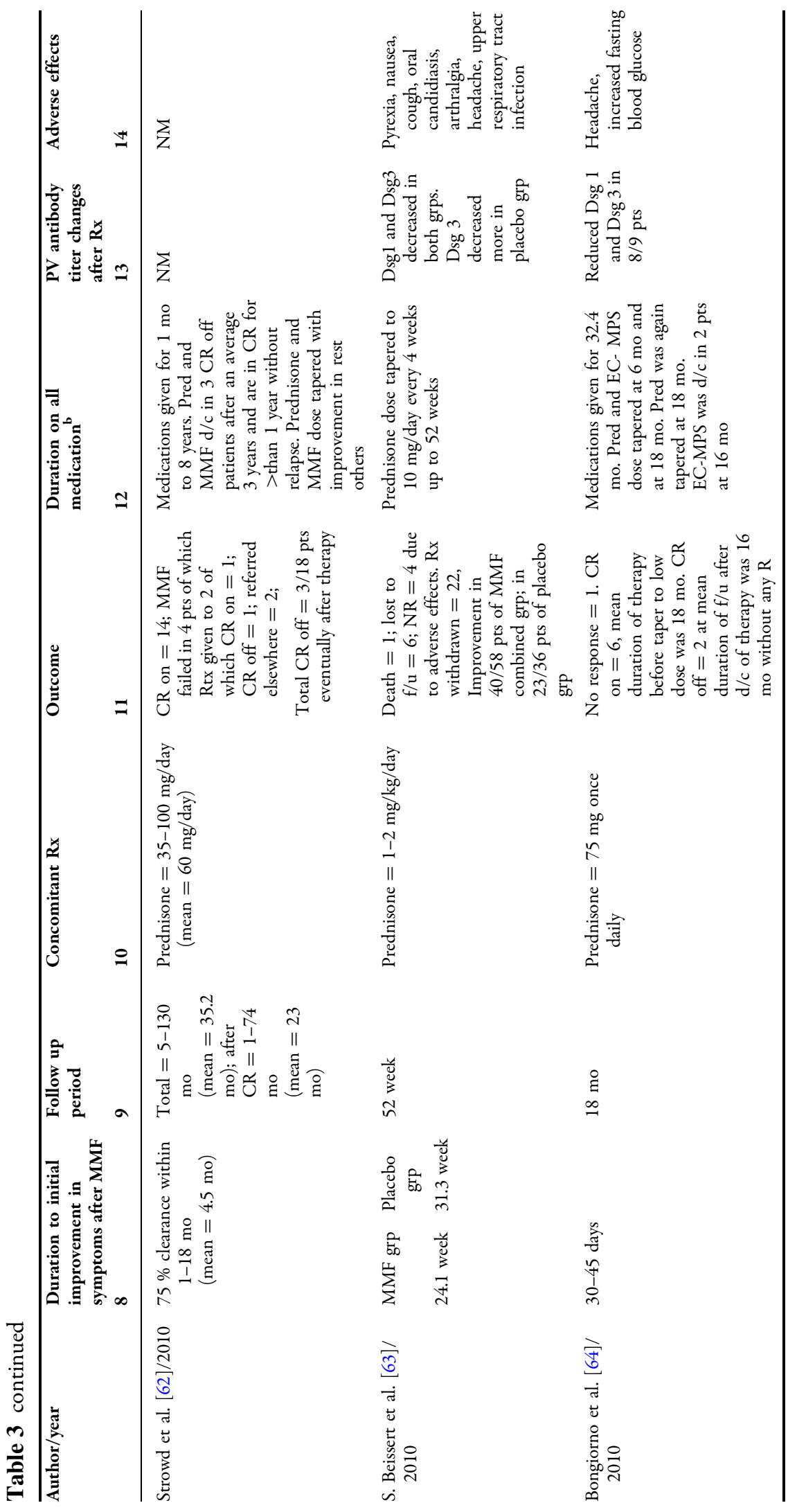




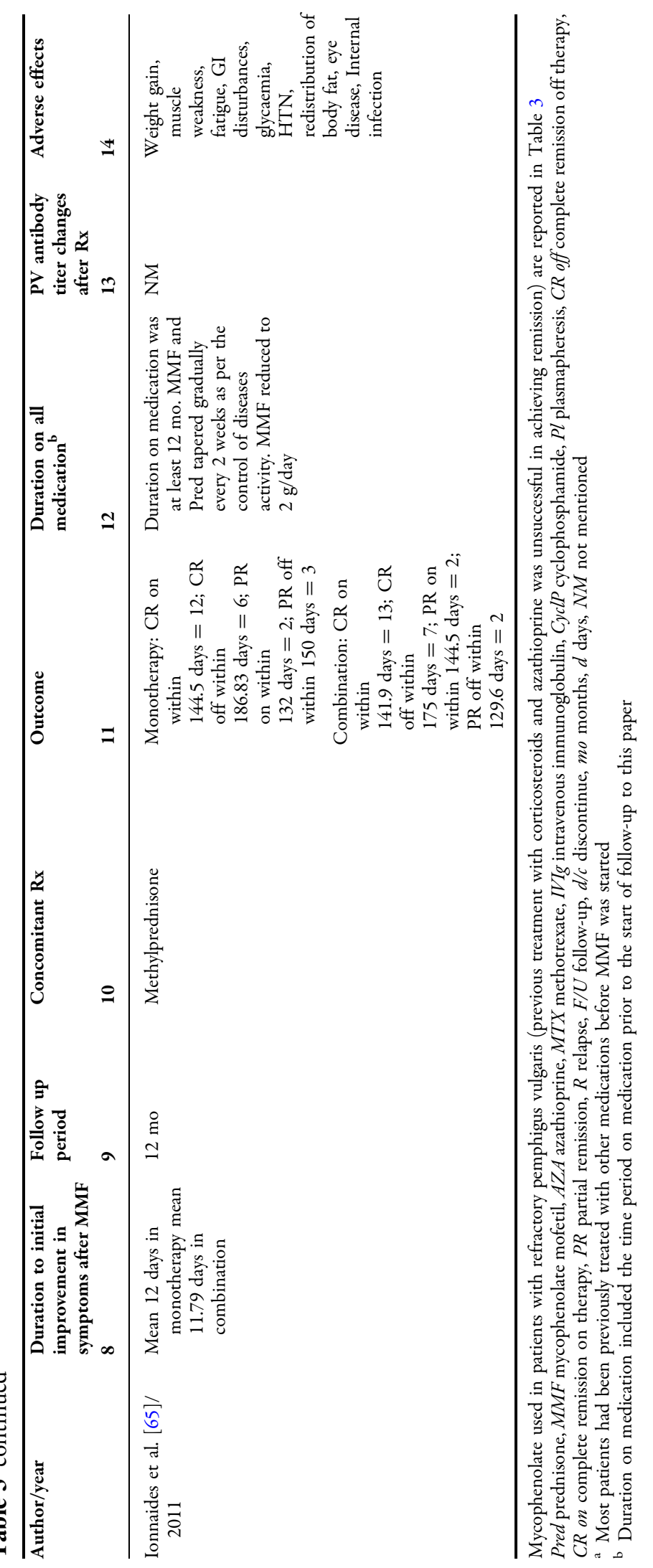




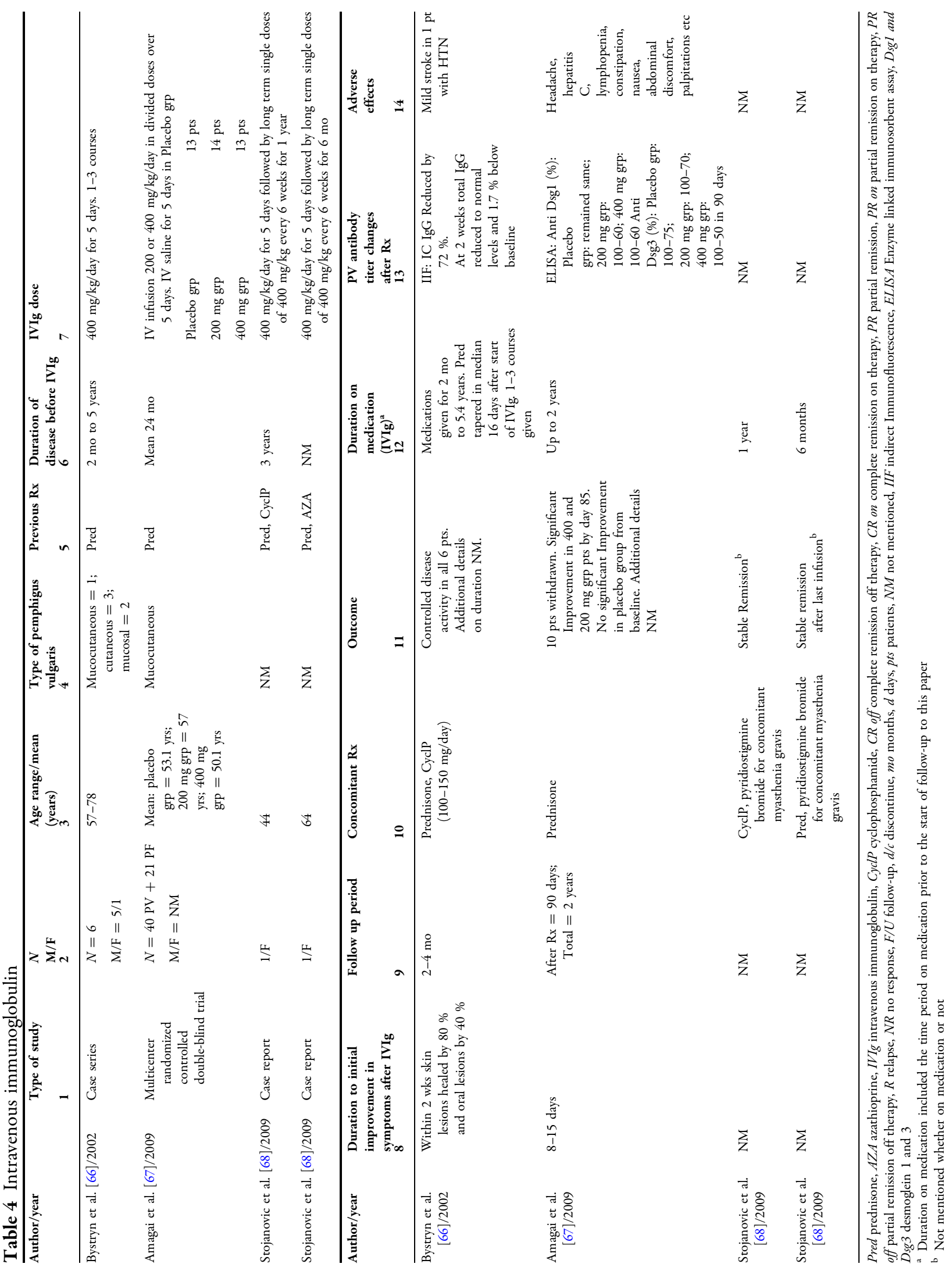




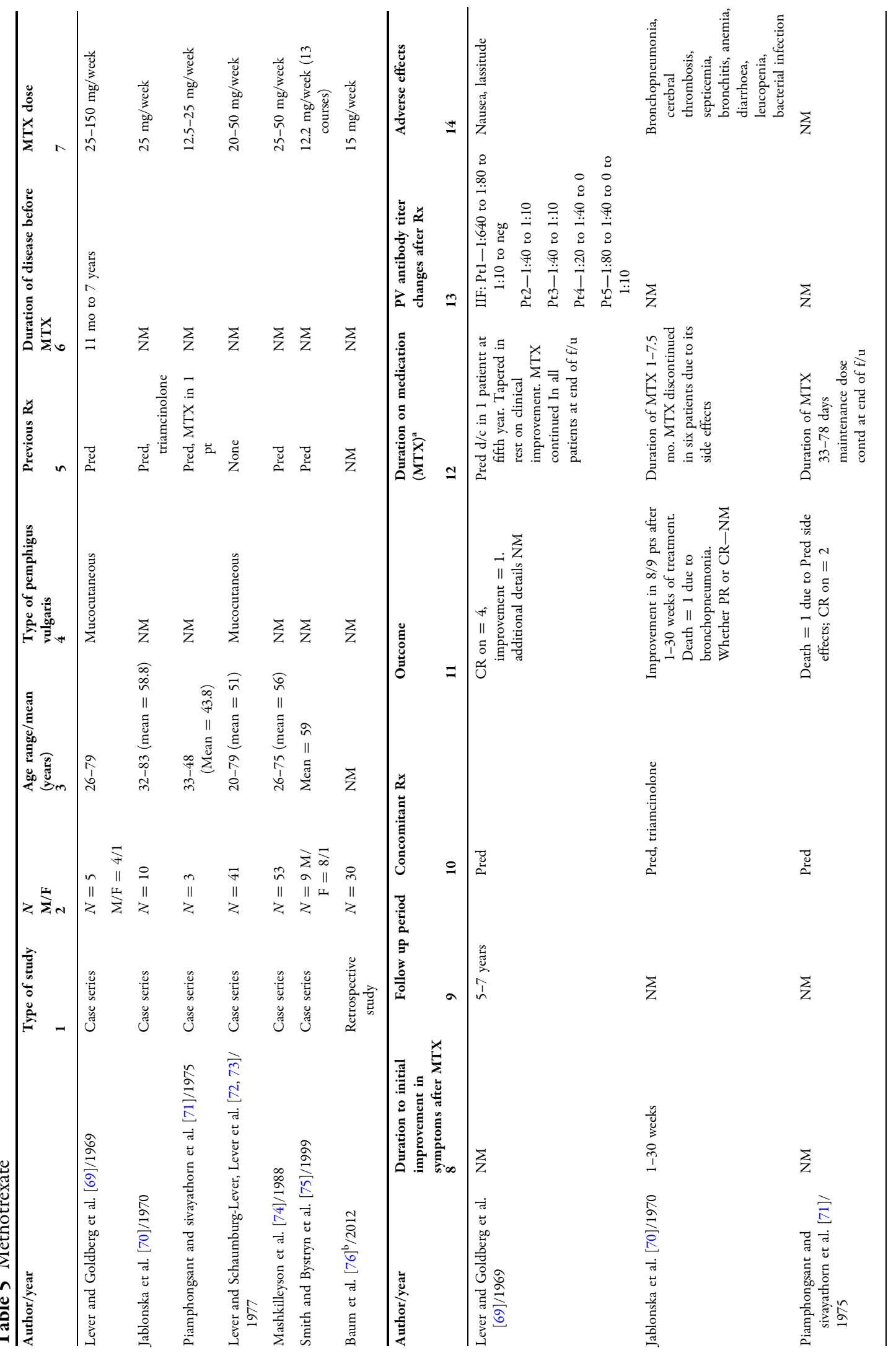




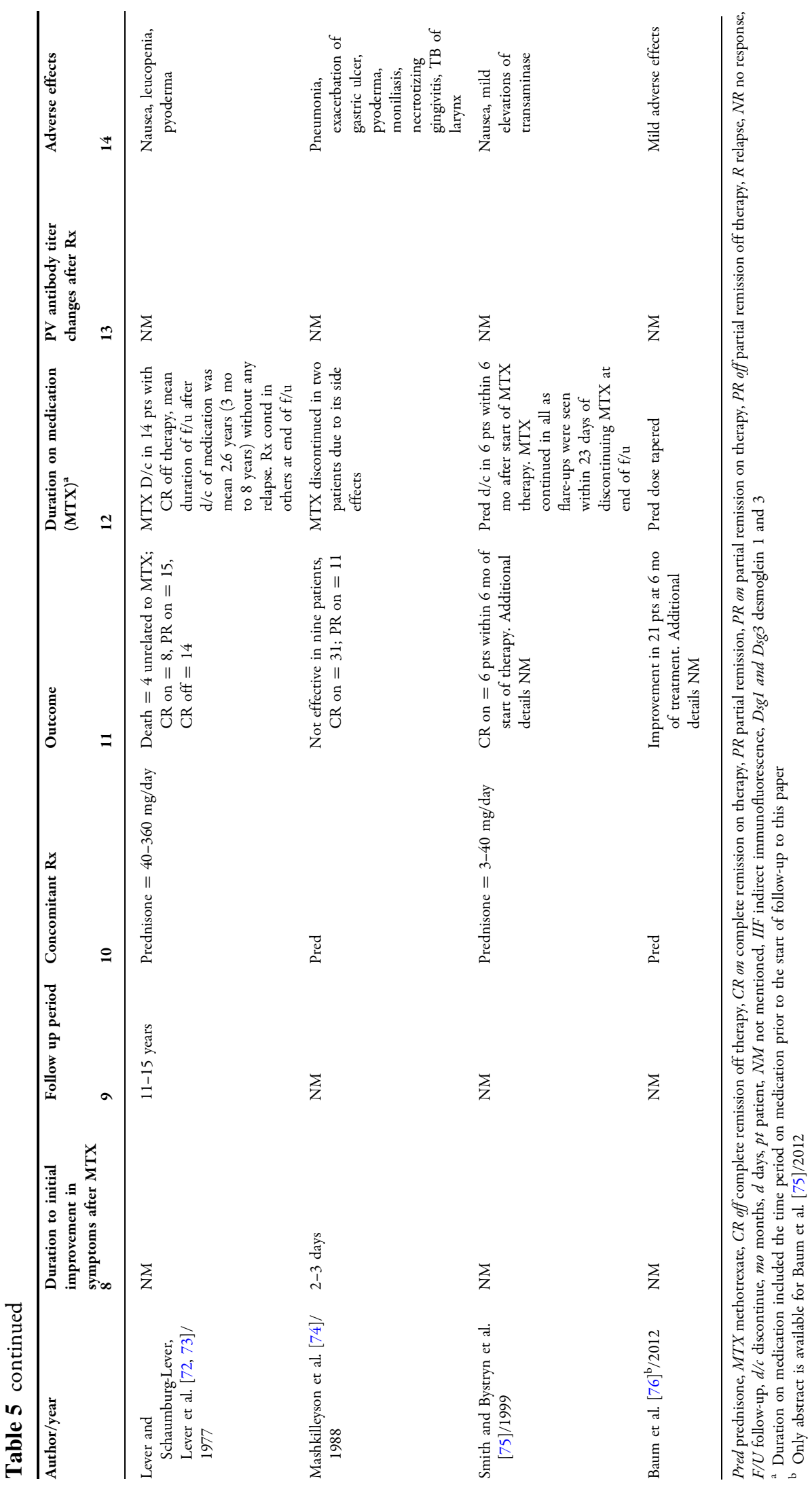




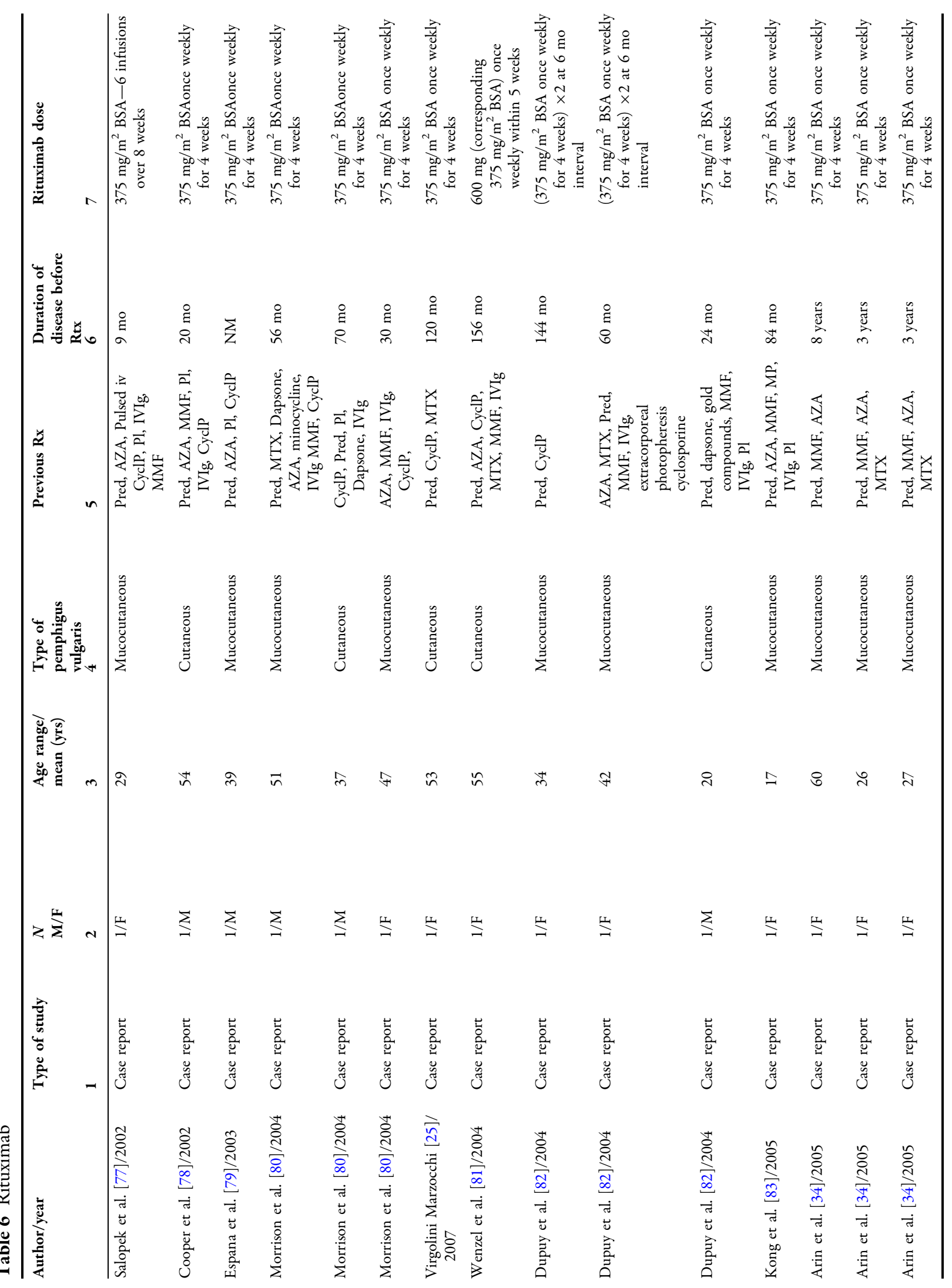




\begin{tabular}{|c|c|c|c|c|c|c|c|c|c|c|c|c|c|c|}
\hline 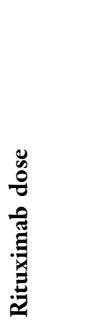 & $n$ & 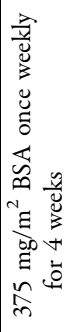 & 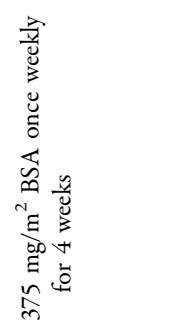 & 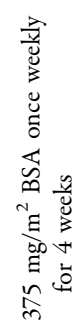 & 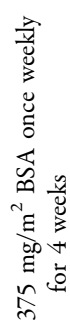 & 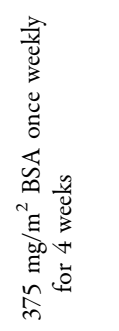 & 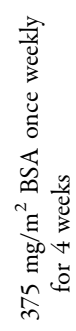 & & & 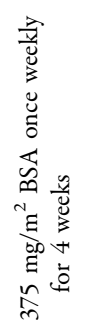 & 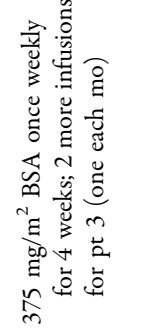 & 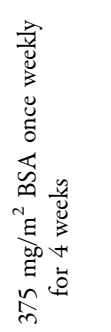 & 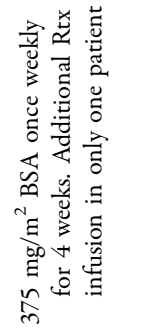 & 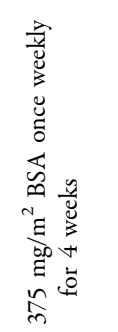 \\
\hline 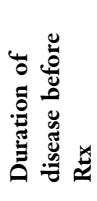 & & 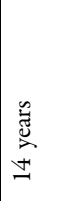 & $\begin{array}{l}\stackrel{\tilde{J}}{\tilde{J}} \\
\stackrel{n}{i} \\
i\end{array}$ & $\begin{array}{l}\stackrel{0}{\Xi} \\
\stackrel{\infty}{\circ}\end{array}$ & $\begin{array}{l}\stackrel{̊}{\Xi} \\
\sigma\end{array}$ & \begin{tabular}{l}
$\stackrel{\bigcirc}{\Xi}$ \\
\multirow{J}{*}{}
\end{tabular} & $\stackrel{̊}{\curvearrowleft}$ & & & 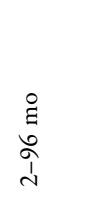 & 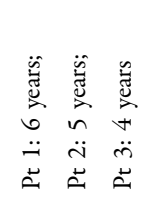 & 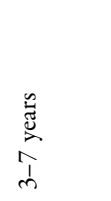 & 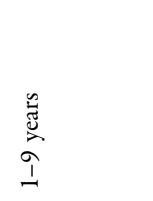 & 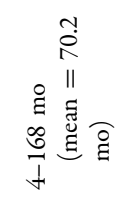 \\
\hline 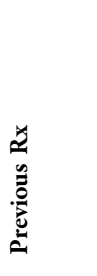 & in & 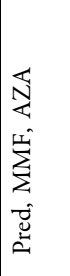 & 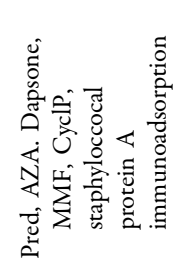 & 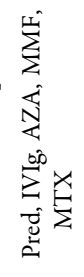 & 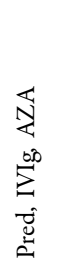 & 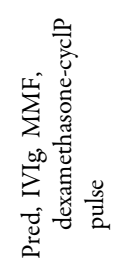 & 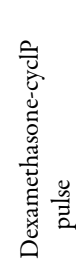 & 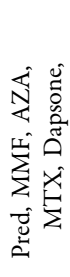 & & 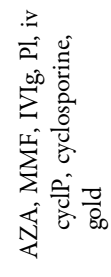 & 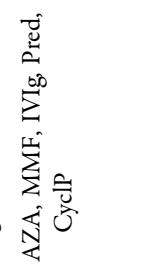 & 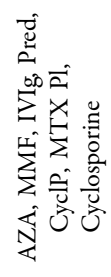 & 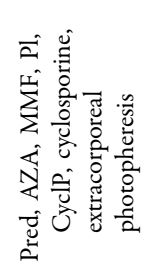 & 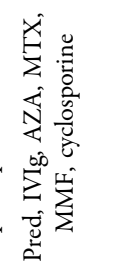 \\
\hline 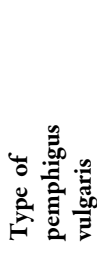 & & 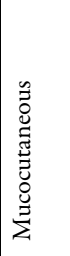 & 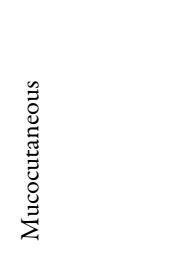 & 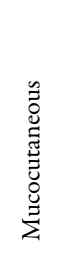 & 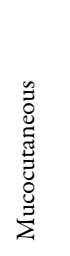 & 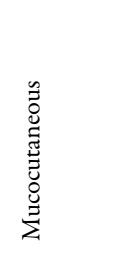 & 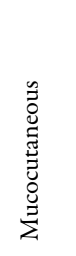 & 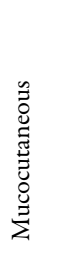 & & 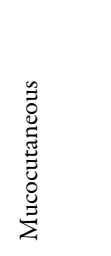 & 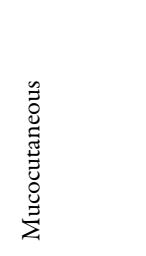 & 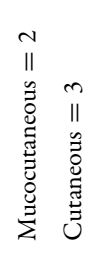 & 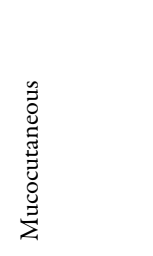 & 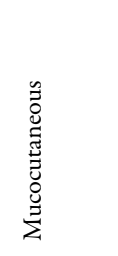 \\
\hline 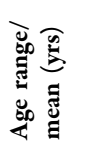 & $\infty$ & in & 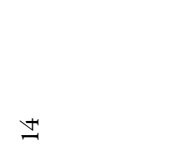 & 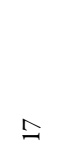 & m & $\stackrel{\infty}{\infty}$ & $\vec{\infty}$ & 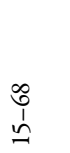 & 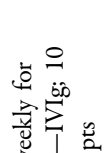 & $\begin{array}{l}\hat{n} \\
\text { तo } \\
\vdots \\
\vdots\end{array}$ & $\begin{array}{lll}\vec{n} & \stackrel{n}{n} \\
\ddot{\Xi} & \ddot{\Xi} & \ddot{\Xi}\end{array}$ & 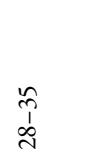 & $\begin{array}{l}\text { Ô. } \\
\stackrel{1}{1}\end{array}$ & 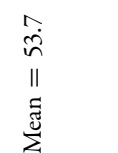 \\
\hline$z \frac{\vec{U}}{2}$ & $N$ & $\stackrel{\Perp}{=}$ & $\Sigma$ & $\stackrel{\Perp}{ \pm}$ & $\stackrel{\Perp}{=}$ & $\stackrel{\Perp}{ \pm}$ & $\stackrel{\Perp}{ \pm}$ & 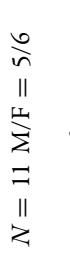 & 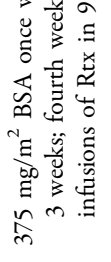 & 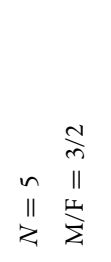 & $\begin{array}{ll} & \vec{\lambda} \\
m & \| \\
\pi & \| \\
\| & \Sigma\end{array}$ & $\begin{array}{ll} & \vec{f} \\
n & \| \\
\| & \| \\
z & \stackrel{\Sigma}{z}\end{array}$ & $\begin{array}{lc} & \frac{n}{n} \\
0 & \\
\| & \| \\
\| & 1 \\
z & \Sigma\end{array}$ & 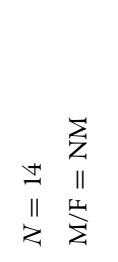 \\
\hline 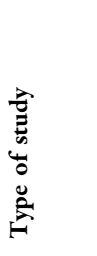 & - & 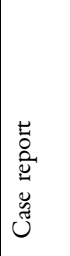 & 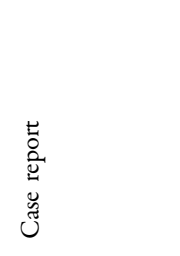 & 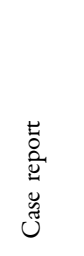 & 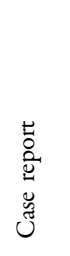 & 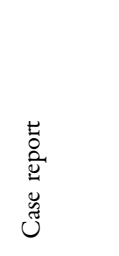 & 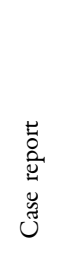 & $\begin{array}{l}\mathscr{y} \\
\tilde{y} \\
\tilde{y} \\
\tilde{U}\end{array}$ & 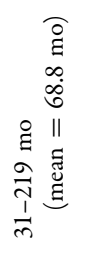 & 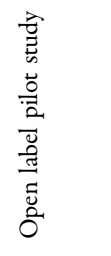 & 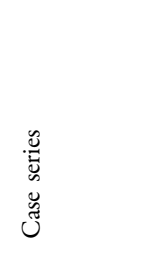 & 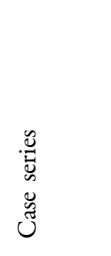 & 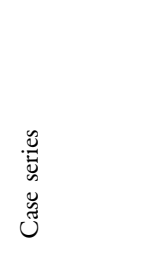 & 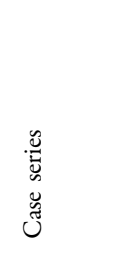 \\
\hline 莬 & & 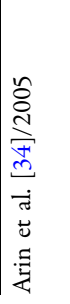 & 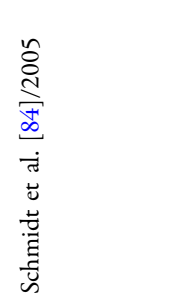 & 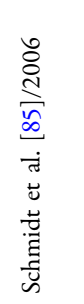 & 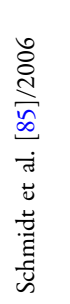 & 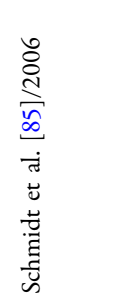 & 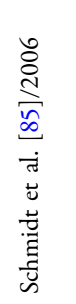 & 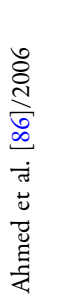 & 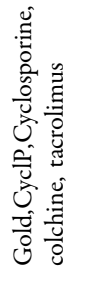 & 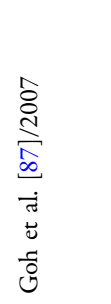 & 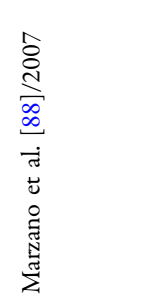 & 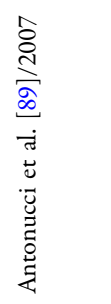 & 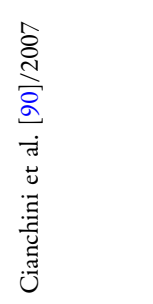 & 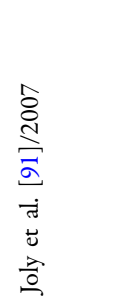 \\
\hline
\end{tabular}




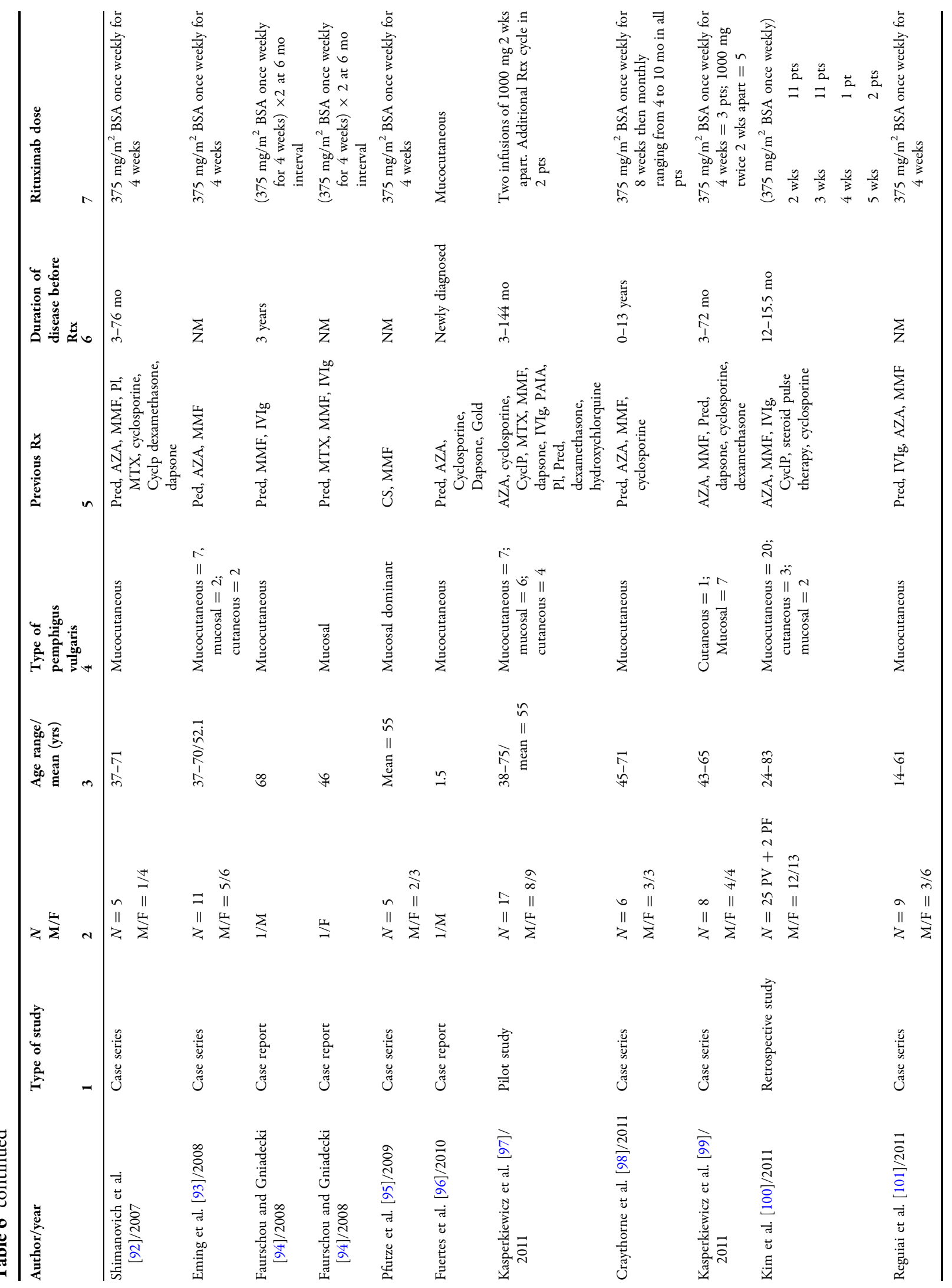




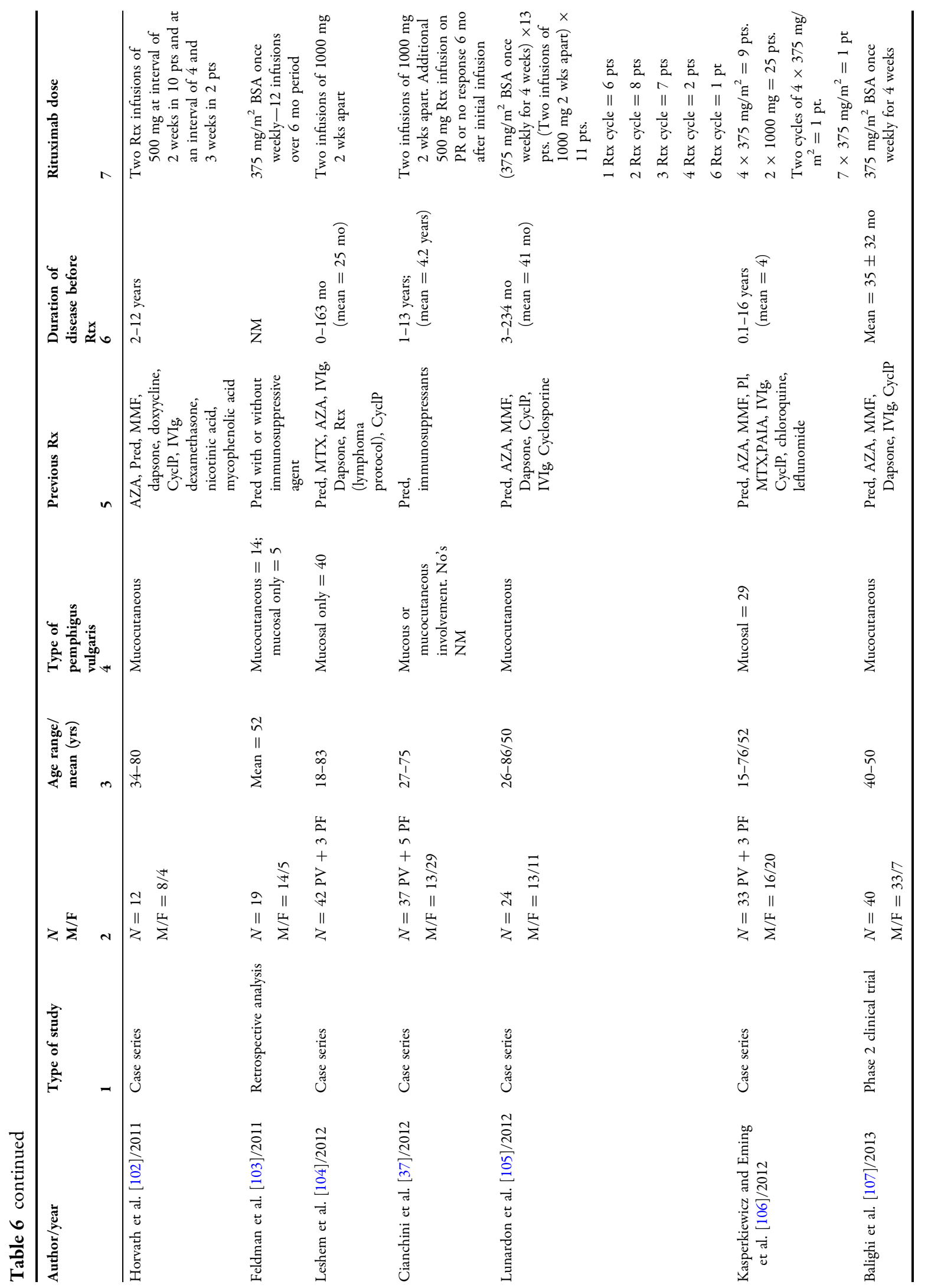




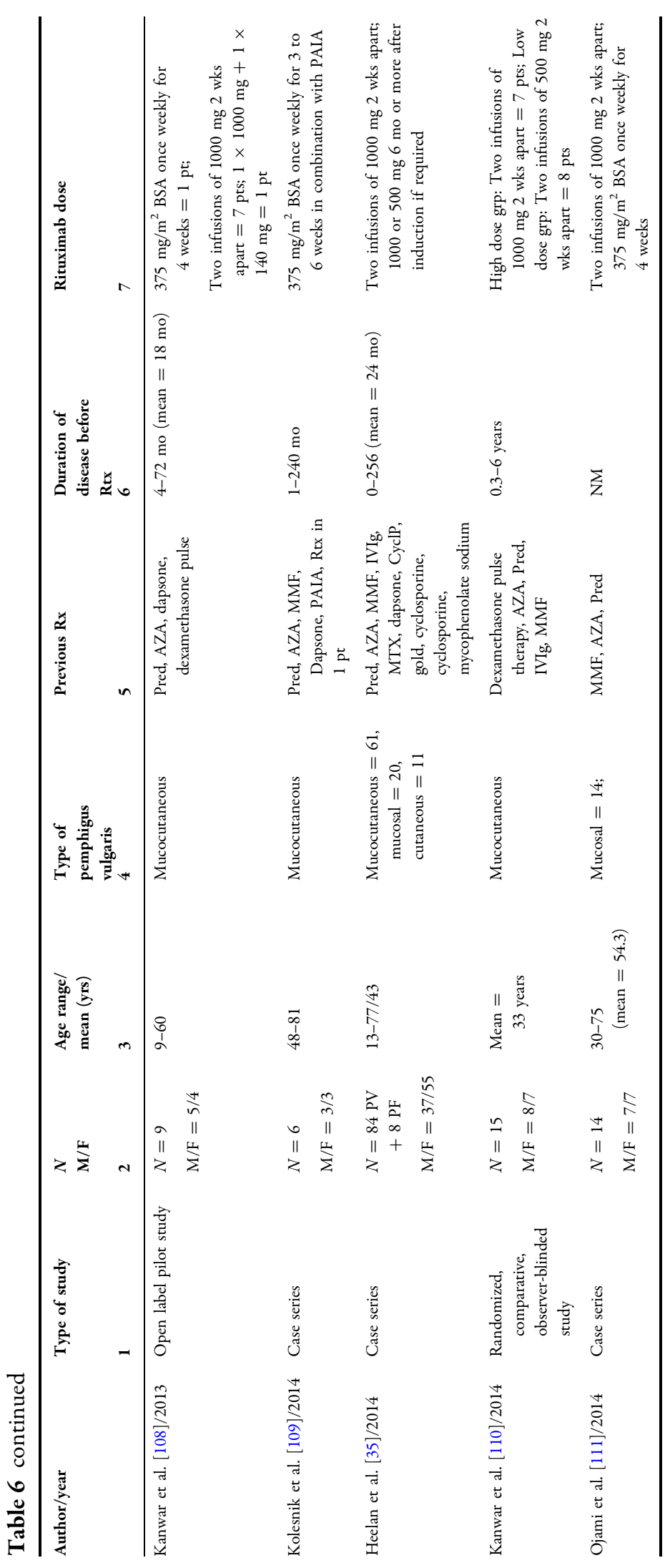




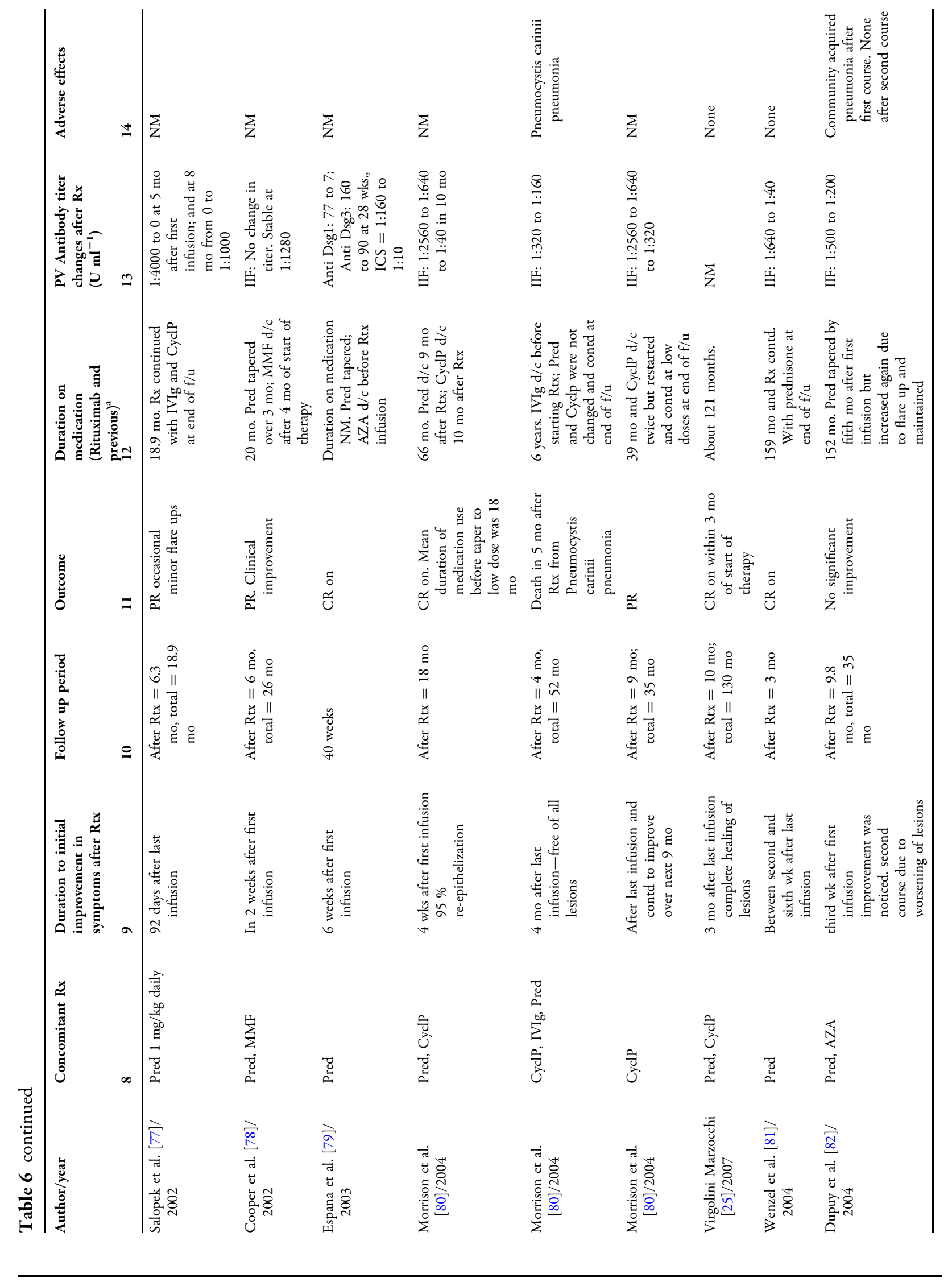




\begin{tabular}{|c|c|c|c|c|c|c|}
\hline 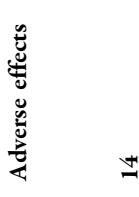 & 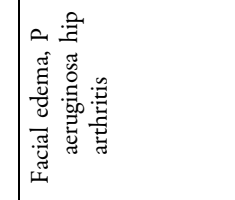 & 芝 & 玄 & 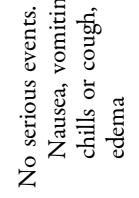 & 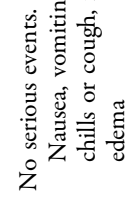 & 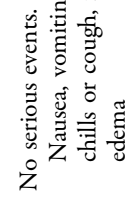 \\
\hline 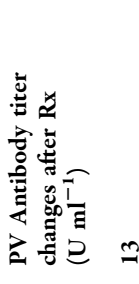 & 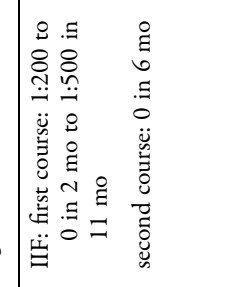 & 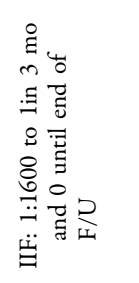 & 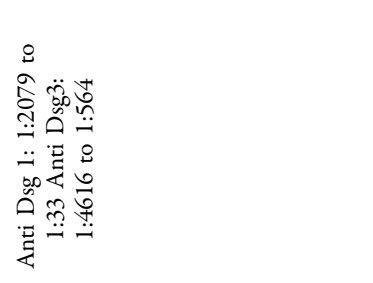 & 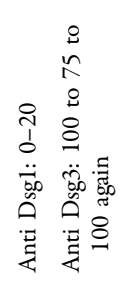 & 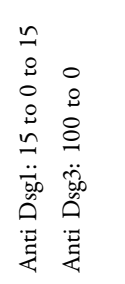 & 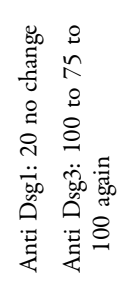 \\
\hline 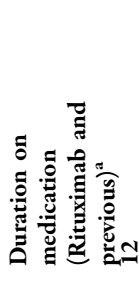 & 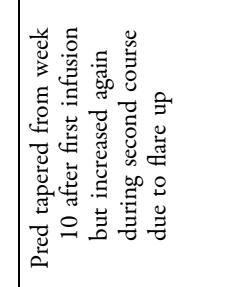 & 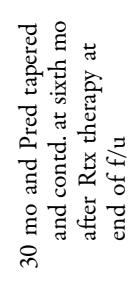 & 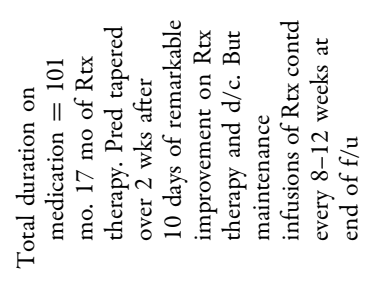 & 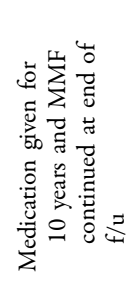 & 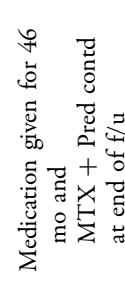 & 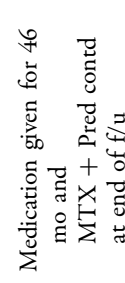 \\
\hline 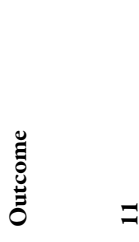 & 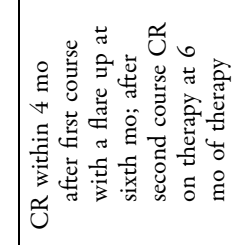 & 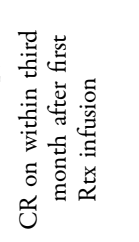 & $\begin{array}{l}\text { जै } \\
\tilde{0}\end{array}$ & $\begin{array}{l}\tilde{0} \\
\tilde{U}\end{array}$ & $\cong$ & $\cong$ \\
\hline 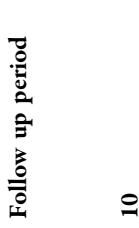 & 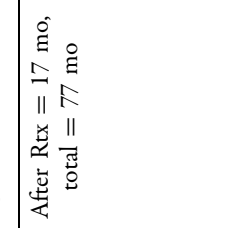 & 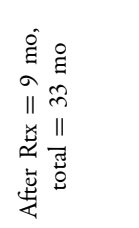 & 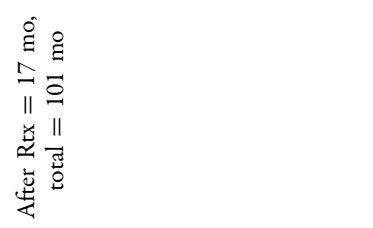 & 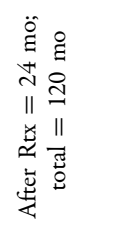 & 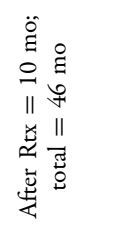 & 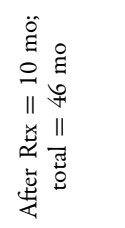 \\
\hline 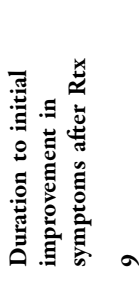 & 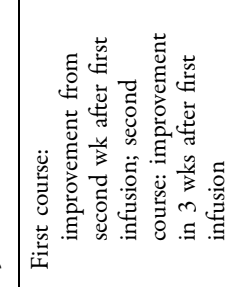 & 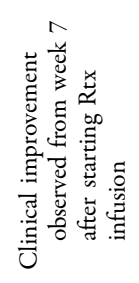 & 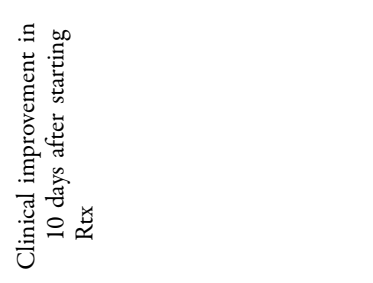 & $\bar{z}$ & $\bar{z}$ & $\sum_{z}$ \\
\hline م & 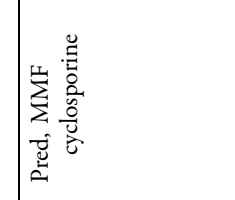 & 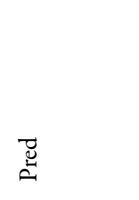 & 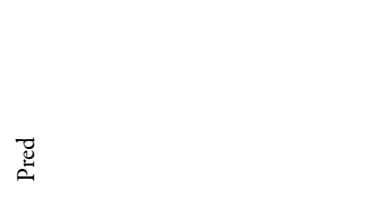 & 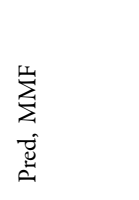 & 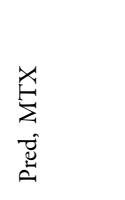 & 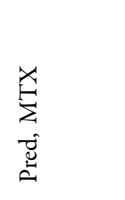 \\
\hline 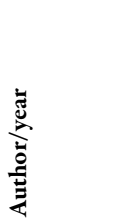 & 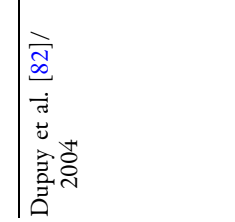 & 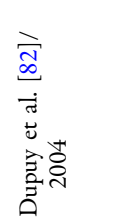 & 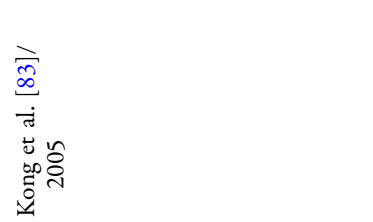 & 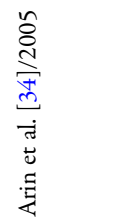 & 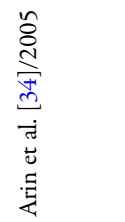 & 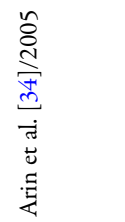 \\
\hline
\end{tabular}




\begin{tabular}{|c|c|c|c|c|c|c|c|c|c|c|}
\hline 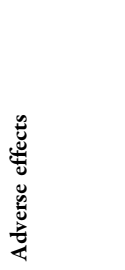 & \pm & 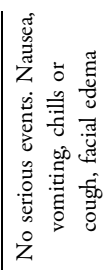 & 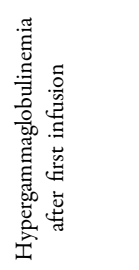 & $\begin{array}{l}\tilde{\tilde{\sigma}} \\
z\end{array}$ & 蒿 & 芩 & 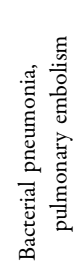 & 䓂 & 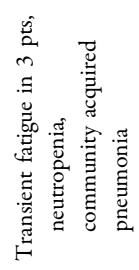 & 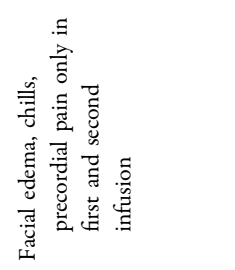 \\
\hline 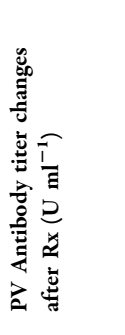 & 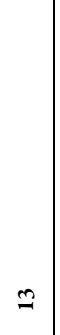 & 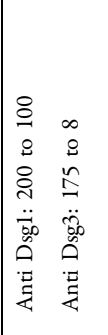 & 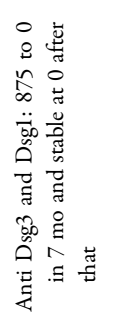 & 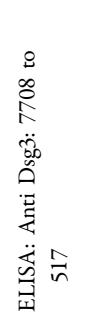 & 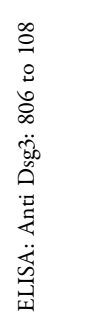 & 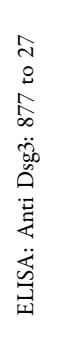 & 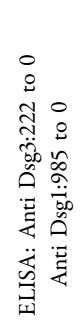 & 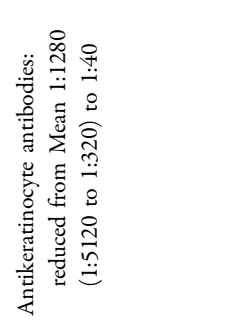 & 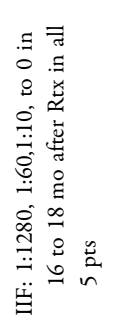 & 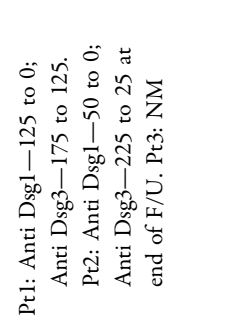 \\
\hline 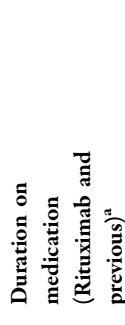 & & 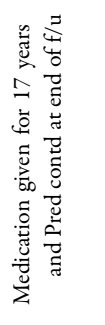 & 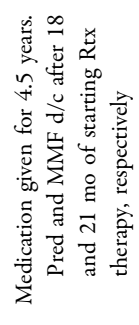 & 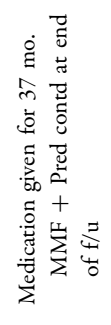 & 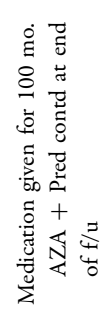 & 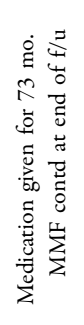 & 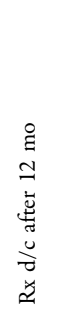 & 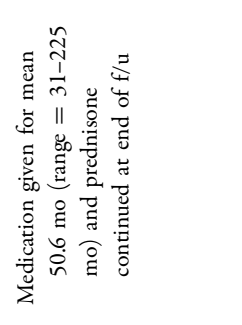 & 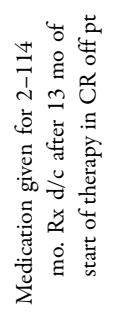 & 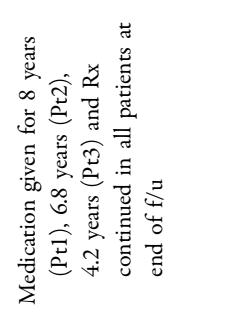 \\
\hline & $=$ & $\begin{array}{l}\tilde{\sigma} \\
\tilde{\tilde{U}}\end{array}$ & $\begin{array}{l}\text { मै } \\
\text { चै }\end{array}$ & $\cong$ & $\cong$ & $\begin{array}{l}\tilde{\delta} \\
\tilde{U}\end{array}$ & 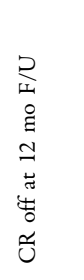 & 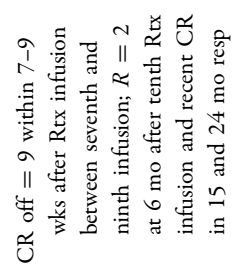 & 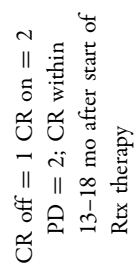 & 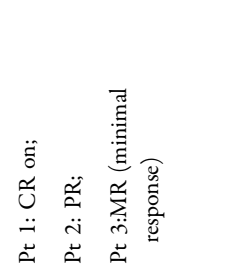 \\
\hline 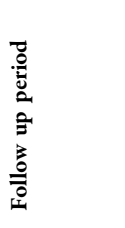 & 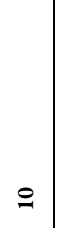 & 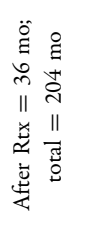 & 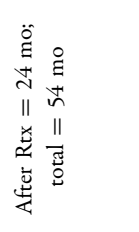 & 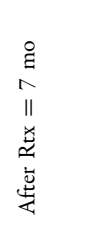 & 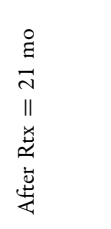 & 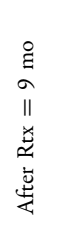 & 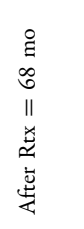 & 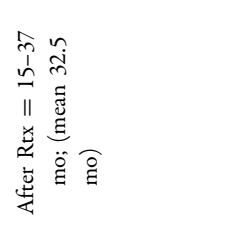 & 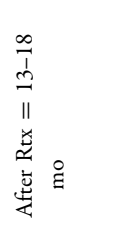 & 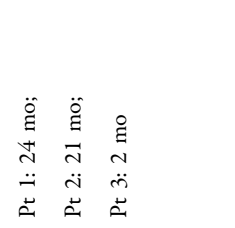 \\
\hline 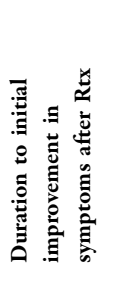 & $a$ & 文 & 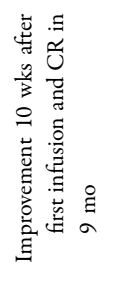 & 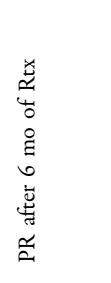 & 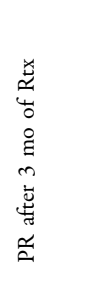 & 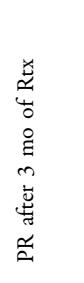 & 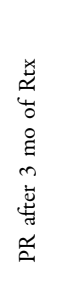 & 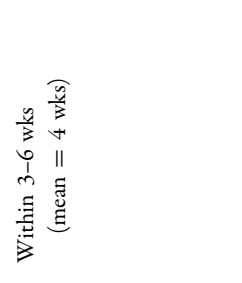 & 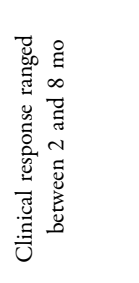 & 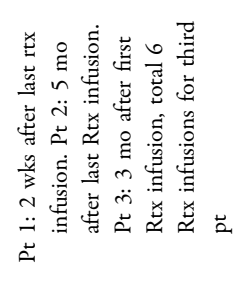 \\
\hline & $\infty$ & 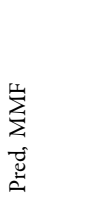 & 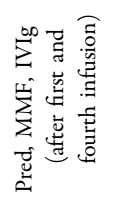 & 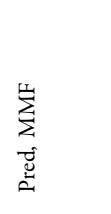 & 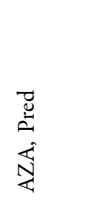 & 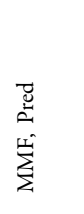 & 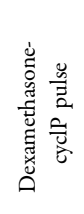 & $\bar{z}$ & 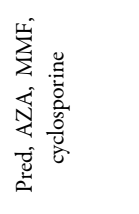 & $\vec{\Xi}$ \\
\hline 节 & & 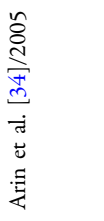 & 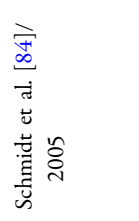 & 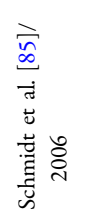 & 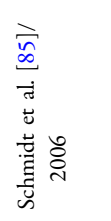 & 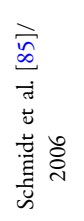 & 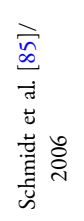 & 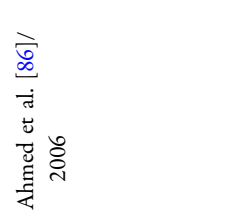 & 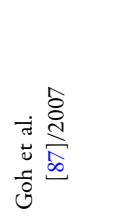 & 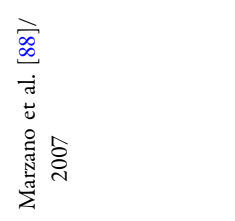 \\
\hline
\end{tabular}




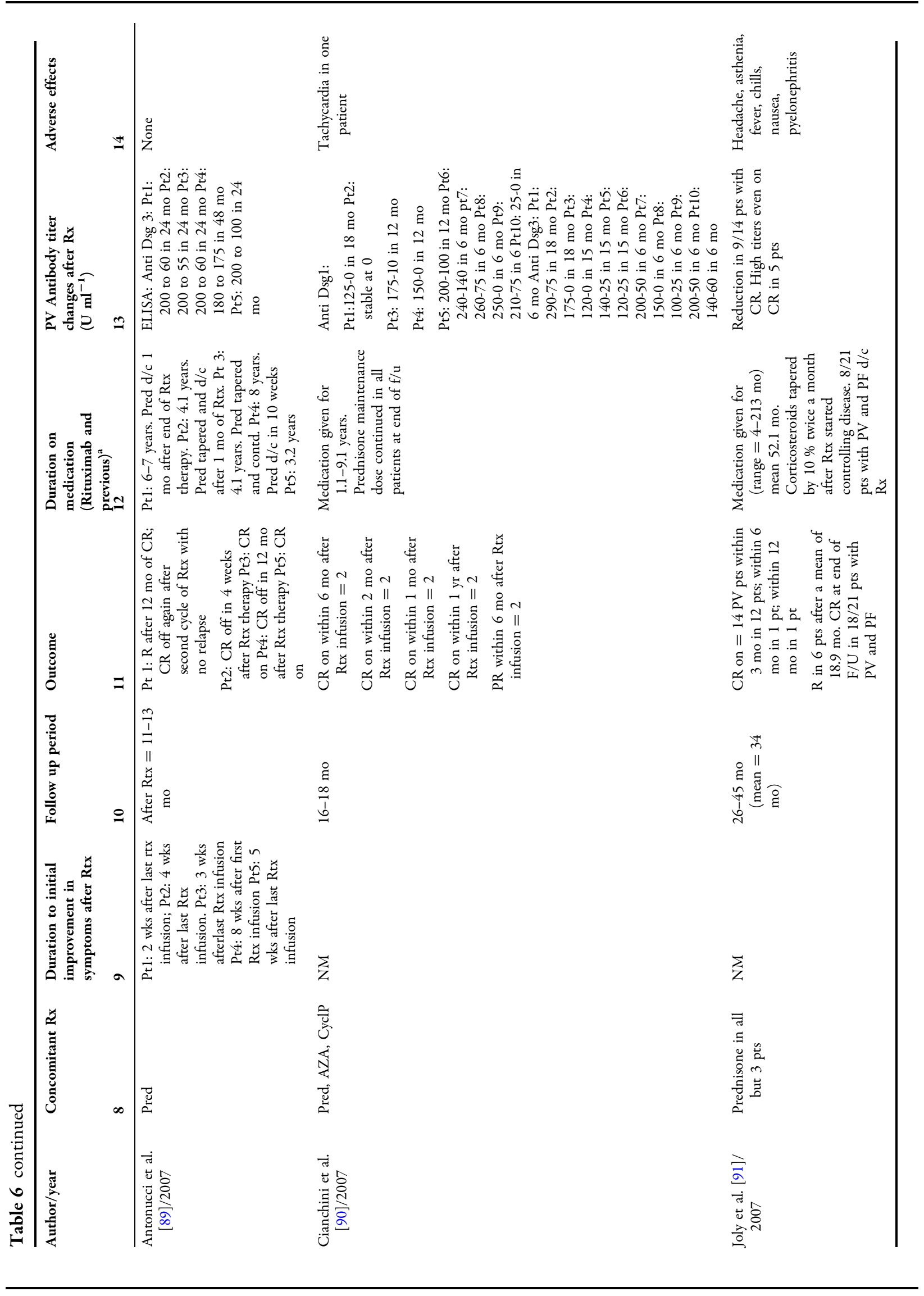




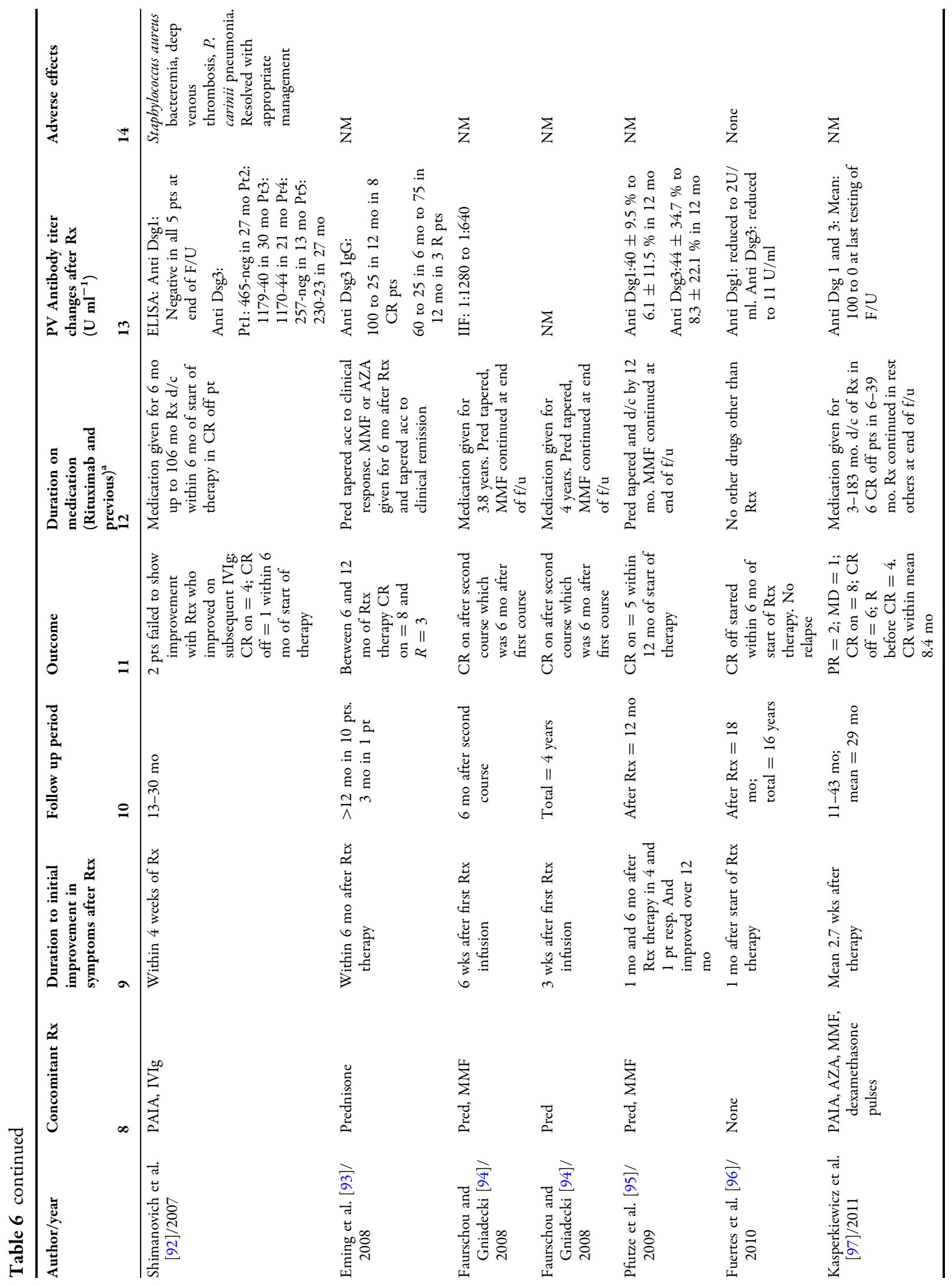




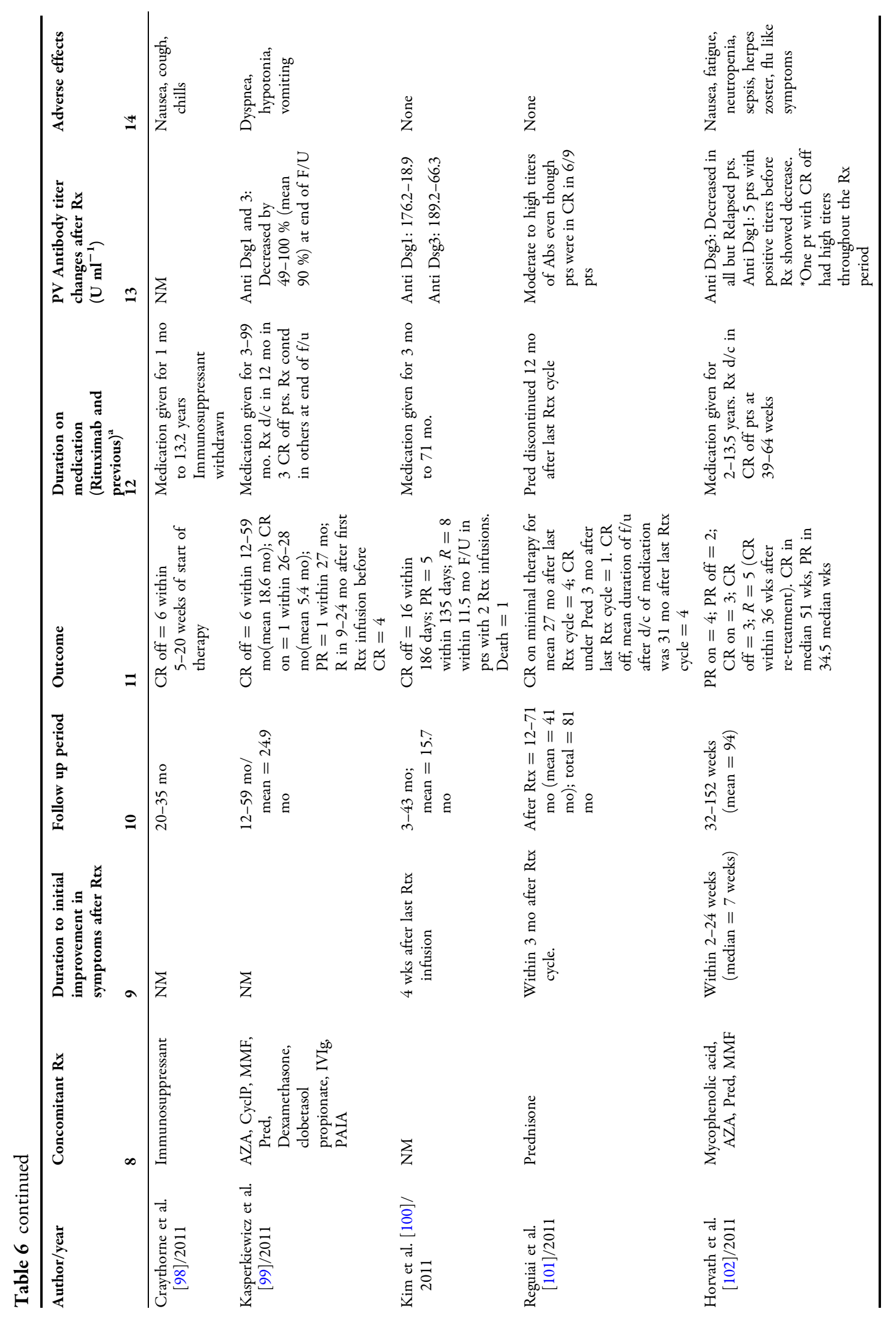




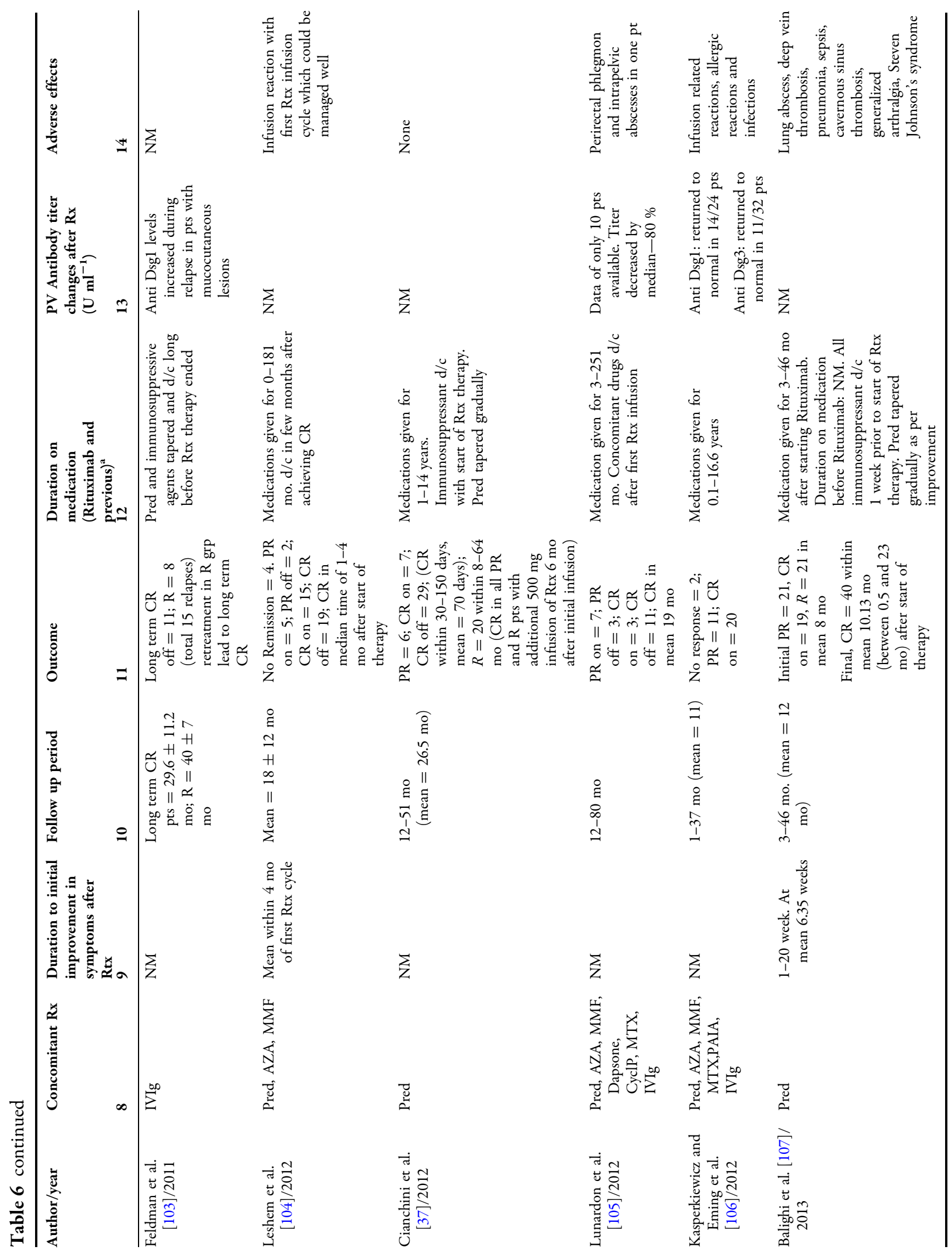




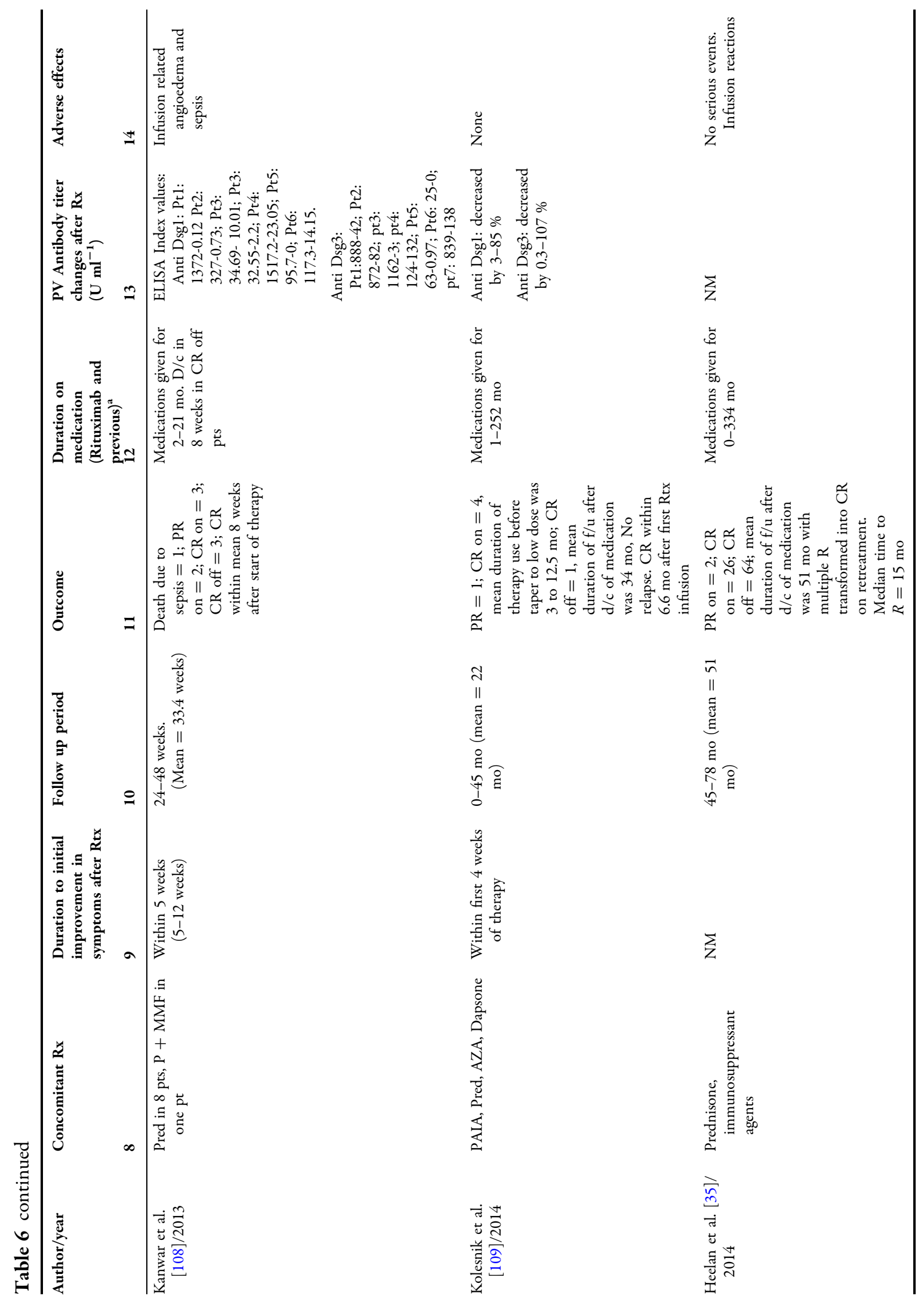




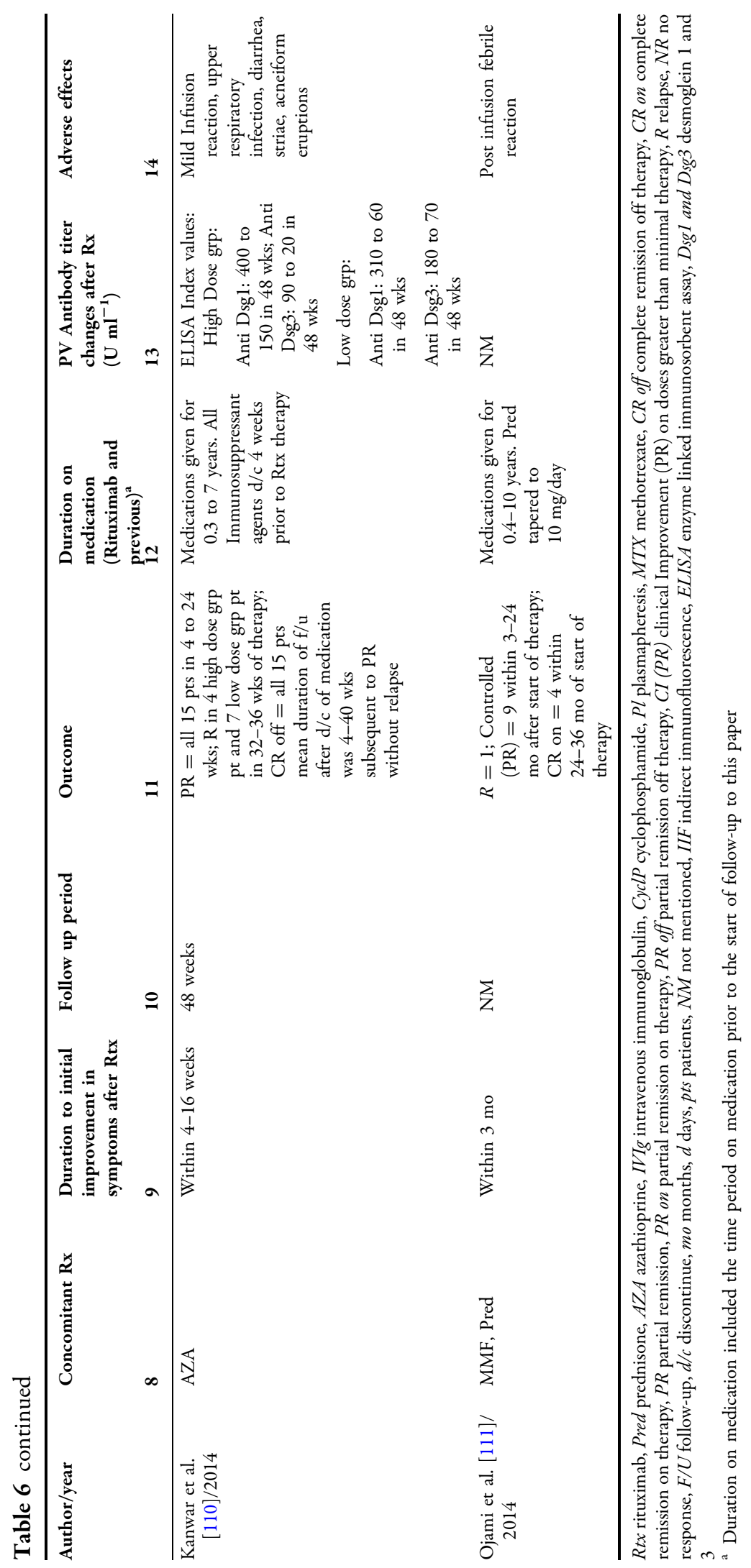




\section{Adverse Effects Reported in Table 2}

Adverse effects in patients on azathioprine and corticosteroids reported in these publications included leukopenia, anemia, thrombocytopenia, pancytopenia, hepatotoxicity, hypertension, gastrointestinal problems, lethargy, weight gain, muscle weakness, adrenal suppression, alopecia, and rash-like skin disorders.

\section{Mycophenolate Mofetil (MMF)}

Mycophenolate Mofetil was approved by the FDA in 1995 as an immunosuppressant to prevent organ transplant rejection.

\section{Mechanism of Action}

After oral administration, mycophenolate is absorbed rapidly and then gets converted to the active metabolite mycophenolic acid (MPA). This active metabolite inhibits inosine monophosphate dehydrogenase selectively and hence inhibits de novo pathway of purine synthesis in $\mathrm{T}$ and $\mathrm{B}$ cells, which results in inhibition of $\mathrm{T}$ and $\mathrm{B}$ cell proliferation [20].

Publications reporting use of MMF as an adjuvant to corticosteroids in PV were included in Table 1. Additional papers which have reported on the use of mycophenolate in patients with refractory PV (previous treatment with corticosteroids and azathioprine was unsuccessful in achieving remission) are summarized in Table 3. Of 31 papers in Table 1, three had included MMF as one of the treatment modalities.

\section{Publication Type, Patient Profiles, and Sample}

\section{Sizes}

The first case series on use of MMF in PV patients was published in 1999.

Four case series were included, with the number of cases included in the individual papers ranging from 9 to 31 cases (a total of 64 patients in four case series); two were case reports describing single patients and two were randomized prospective trials $(n=94$ and $n=21$, respectively). One additional randomized clinical trial enrolled both PV and PF patients $[n=36(\mathrm{PV})+11(\mathrm{PF})$; results were not reported separately for the $\mathrm{PV}$ and $\mathrm{PF}$ patients in this study] and one retrospective analysis $(n=18)$ is summarized in the tables. The total number of patients treated with MMF in these 10 reports was 247 .

Age at initial diagnosis of $\mathrm{PV}$ in these publications ranged from 6 to 78 years.

\section{Medication Use and Duration of PV Before MMF Was Started}

Medication use and duration of PV before MMF was started ranged from 1 month to 14 years. During this period patients were on a combination of corticosteroids and azathioprine. At the time mycophenolate was added, the azathioprine was discontinued; however, the patients continued to be on corticosteroids. One publication (Powell et al.) reported on patients in whom multiple medications like methotrexate, cyclophosphamide, IVIg, dapsone, gold, thalidomide, and minocycline along with azathioprine and corticosteroids were tried prior to addition of mycophenolate [21].

The starting dosage of mycophenolate mofetil used was 2-3 g/day in all reports.

\section{Duration of Follow-up}

Duration of clinical follow-up of the individual patients after the start of MMF therapy ranged from 5 to 130 months.

\section{Duration Before Any Clinical Improvement Was Noted}

First improvement in lesions was noted after 2-24 weeks after addition of mycophenolate to the existing medication regimen. 


\section{Duration to Complete Remission (On and Off} Therapy) After Addition of MMF

Duration to complete remission on therapy was reported in six articles and, ranged from 2 to 16 months, in 104 patients.

Duration to complete remission off therapy was reported in one article and, ranged from 24 to 36 months, in 17 patients.

\section{Remission}

Of a total of 247 patients, 104 patients achieved complete remission on therapy and 17 patients achieved complete remission off therapy. A total of 76 patients achieved partial remission, and the duration to achieve that ranged from 129 to 150 days after the start of therapy. Failure of MMF was mentioned in four reports $(N=176)$ in 18 patients who were referred for treatment with rituximab or IVIg. Two patients were still being treated at the time of publication, 29 patients were lost to follow-up or withdrawn from study, and death occurred in one patient.

\section{Adverse Effects}

Adverse effects in patients on mycophenolate and corticosteroids reported in these publications included gastrointestinal problems, myalgia, neutropenia, and lymphopenia, which were the most common side effects reported. Headache, increased fasting blood glucose level, and hypertension, nausea, depression, pyrexia, redistribution of body fat, eye disease, weight gain, fatigue, and arthralgia were also reported.

In the one publication where enteric coated mycophenolate sodium was used, the side effects reported were headache and increased fasting blood glucose level.

\section{Intravenous Immunoglobulin (IVIg)}

IVIg was approved by the FDA for primary immune deficiency in 1952 [22].

\section{Mechanism of Action}

Intravenous immunoglobulins (IVIg) are obtained from a plasma pool of thousands of donors [22].

These immunoglobulins neutralize and slow down the production of circulating pemphigus antibodies [23].

\section{Publication Type, Patient Profiles, and Sample Sizes}

The studies reporting use of IVIg in PV are summarized in Table 4 . The first case series on IVIg in PV was published in 2002.

One case series $(n=6)$, two case reports describing single patients, and one randomized placebo-controlled double-blind trial $(n=40)$ are summarized in Table 4 , with a total of 48 patients included in these four papers. These reports included patients previously treated with corticosteroids, cyclophosphamide, azathioprine, and methotrexate without adequate response, prior to start of IVIg.

Age at initial diagnosis of $\mathrm{PV}$ in these publications ranged from 41 to 78 years.

\section{Medication Use}

The dosage of IVIg used was $400 \mathrm{mg} / \mathrm{kg} /$ day for 5 days followed by long- or short-term single doses of $400 \mathrm{mg} / \mathrm{kg} /$ day every 6 weeks for 6 months to 1 year. Concomitant drugs mainly used were corticosteroids in the published studies.

\section{Duration of PV Before IVIg Was Started}

This ranged from 2 months to 5 years.

\section{Duration of Total Follow-up}

Duration of total clinical follow-up of the individual patients ranged from 2 months to 2 years. 


\section{Duration Before Any Clinical Improvement Was Noted}

First improvement in lesions was reported within 2-3 weeks of first IVIg infusion in all 48 patients.

\section{Duration to Start of Taper of Corticosteroids}

Only one case series of six patients described the duration to the start of taper of corticosteroids and only mentioned that the median time was 16 days after the start of IVIg infusions.

\section{Duration to Complete Remission (On and Off Therapy)}

This information was not available from the publications. However, all reports discussed improvement in all patients treated with IVIg; in six patients this was achieved within 3 weeks and in 29 patients within 3-12 months. Thirteen patients in the placebo group had no improvement.

\section{Adverse Effects in Patients on IVIg Reported} in Table 4

Headache, abdominal discomfort, nausea, constipation, lymphopenia, hepatitis C, and palpitations.

\section{Methotrexate}

Methotrexate was approved by the FDA for psoriasis in 1971 and for rheumatoid arthritis in 1988.

\section{Mechanism of Action}

Methotrexate inhibits the metabolism of folic acid and is used as a chemotherapeutic and immunosuppressive agent. Methotrexate allosterically inhibits dihydrofolate reductase, which plays a role in tetrahydrofolate synthesis. As folic acid is essential for normal cell growth and replication, methotrexate is effective against malignant cell growth and has anti-inflammatory effects [24].
Publication Type, Patient Profiles, and Sample Sizes

The studies reporting use of methotrexate in PV are summarized in Table 5. The first case series on MTX in PV was published in 1969.

Publications reporting use of methotrexate in PV were included in Table 1 ( 7 of 31 papers included methotrexate), and additional papers that reported on the use of methotrexate as the initial adjunctive treatment to corticosteroids are summarized in Table 5.

Six case series were included, with the number of cases included in the individual papers ranging from 3 to 53 cases (total of 121 patients in six case series), and one retrospective cohort study $(n=30)$ are summarized in the tables. In all, a total of 151 patients treated with MTX are reported in seven studies.

Age at initial diagnosis of $\mathrm{PV}$ in these publications ranged from 20 to 83 years.

\section{Medication Use}

The dosage of MTX used in these publications ranged from 12.5 to $150 \mathrm{mg} /$ week. Concomitant drug used along with methotrexate was prednisone.

\section{Duration of PV Before Methotrexate Was Started}

This ranged from 11 months to 7 years.

\section{Duration of Follow-up}

Duration of clinical follow-up of the individual patients after the start of MTX ranged from 5 to 15 years.

\section{Duration Before Any Clinical Improvement} Was Noted

First improvement in lesions was reported within 1-30 weeks after the start of methotrexate therapy. 


\section{Duration to Complete Remission (On and Off}

\section{Therapy)}

Duration to complete remission on therapy was reported in six articles and, ranged from 1 to 30 weeks, in 51 patients.

Duration to complete remission off therapy was reported in one article and, ranged from 3 months to 8 years, in 14 patients.

\section{Remission}

Of a total of 151 patients, at the end of follow-up, 56 patients had achieved partial remission and the duration to achieve that was within 6 months after the start of MTX therapy; 51 patients had achieved complete remission on therapy; and 14 patients had achieved complete remission off therapy. Twelve patients were lost to follow-up. Treatment was not effective in nine patients. Death unrelated to MTX occurred in six patients.

\section{Adverse Effects in Patients on MTX Reported in Table 5}

Nausea, leukopenia, GI upset, fatigue, bacterial infection, bronchopneumonia, septicemia, necrotizing gingivitis, diarrhea, and pyoderma.

\section{Rituximab}

Rituximab was approved in 1997 by the FDA to treat B cell non-Hodgkin lymphoma and in 2006 to treat rheumatoid arthritis.

\section{Mechanism of Action}

Rituximab is a human-mouse chimeric monoclonal antibody to CD20 antigen on B cells. CD20 is a membrane protein that is involved in activation and proliferation of $\mathrm{B}$ cell [25].
Publication Type, Patient Profiles, and Sample Sizes

The studies reporting use of rituximab in PV are summarized in Table 6 . The first case series on PV treated by rituximab was published in 2002 .

Publications which have reported on the use of rituximab in patients with refractory PV (previous treatment with corticosteroids, azathioprine, methotrexate, mycophenolate, IVIg, and cyclophosphamide were unsuccessful in achieving remission) are summarized in Table 6.

Nineteen case series were included, with the number of cases included in the individual papers ranging from 3 to 84 cases (total of 339 patients in 19 case series), 24 were case reports describing single patients, three open label pilot studies $(n=5, n=9$, and $n=17$ ), one randomized prospective trial $(n=15)$, two retrospective analysis $(n=25$ and $n=19)$, and one phase 2 clinical trial $(n=40)$ are summarized in the tables. In all, a total of 493 patients were treated with rituximab.

Age of patients treated with rituximab for PV in these publications ranged from 15 to 86 .

\section{Medication Use}

The dosage of rituximab used was $375 \mathrm{mg} / \mathrm{m}^{2}$ body surface area (BSA) once weekly for 4 weeks or two infusions of $1000 \mathrm{mg}$ at 2 weeks apart. Previously failed treatments before rituximab were prednisone, MMF, AZA, IVIg, MTX, dapsone, CyclP, plasmapheresis, protein A immunoadsorption, cyclosporine, dexamethasone, and gold. Concomitant drug used was prednisone, MMF, AZA, and IVIg.

\section{Duration of PV Before Rituximab Was Started} This ranged from 1 months to 23 years. 


\section{Duration of Follow-up}

Duration of clinical follow-up of the individual patients after the start of rituximab therapy ranged from 6 to 80 months.

\section{Duration Before Any Clinical Improvement Was Noted}

First improvement in lesions was reported within 2 weeks to 8 months after the first rituximab infusion.

\section{Duration to Complete Remission (On and Off} Therapy)

Duration to complete remission on therapy was reported in 32 articles and, ranged from 1 to 36 months, in 184 patients.

Duration to complete remission off therapy was reported in 22 articles and, ranged from 2 to 59 months, in 229 patients.

\section{Remission}

Of a total of 493 patients reported in Table 6, at the end of follow-up, 80 patients had achieved partial remission, and duration to achieve that ranged from 3 to 27 months; 184 patients achieved complete remission on therapy; and 229 patients achieved complete remission off therapy. Death due to sepsis occurred in three patients. Relapses were seen in nine patients. No response to rituximab was seen in 11 patients. However, these patients had response after addition of IVIg or additional cycles of rituximab.

\section{Adverse Effects in Patients on Rituximab Reported in Table 6}

Local pain, nausea, cough, chills, sepsis, and angioedema related to infusion.

\section{OTHER MEDICATIONS}

\section{Other Less Commonly Used Adjuvants from Studies Listed in Table 1}

Gold salts These are widely used in treatment of rheumatoid arthritis. Their action is related to their $\mathrm{T}$ cell-mediated immunosuppressive properties [23].

Plasmapheresis This is used for removing antibodies from the circulation. Reduction in antibodies triggers production of new antibodies as a result of a feedback mechanism [23].

Immunoadsorption With plasmapheresis protective immunoglobulins, albumin, and clotting factors are removed along with harmful pemphigus antibodies. Immunoadsorption selectively traps the harmful pemphigus antibodies through the sulfhydryl filtering membrane. Thus, protective antibodies and plasma components are returned [23].

Cyclophosphamide It has been widely used in the treatment of cancer and also as an immunosuppressant. This drug is converted in the liver to its active metabolites aldophosphamide and phosphoramide mustard. These bind to DNA and inhibit its replication, which leads to cell death. It can be given orally as well as intravenously. One report described cyclophosphamide use in seven patients for treating PV in combination with corticosteroids and azathioprine [26].

Nicotinamide and tetracycline These were used as steroid-sparing agent in combination with corticosteroids and azathioprine in one study of six patients with PV. Their mechanism of action is unclear [27]. 


\section{DISCUSSION}

In this paper, we have summarized the published literature on the management of PV. The published papers were mostly case reports, case series, observational studies, and only eight randomized controlled trials.

As a result of the relative rarity of pemphigus, published randomized trials are limited, which makes it difficult to evaluate the efficacy of different treatment regimens in this disease. This also precludes conduct of a meta-analysis. A Cochrane review published in 2009 concluded that "there is inadequate information available at present to ascertain the optimal therapy for pemphigus vulgaris" [28]. While this remains the case, a summary of the literature provides information on disease course and prognosis as well as medication options, treatment responses, and side effects, which are of relevance to clinicians who treat this disease and patients who suffer from it.

The treatment options for PV have increased over the years. The early publications from the 1970s reported use of high corticosteroid doses over prolonged intervals and significant associated side effects. Later reports on PV management described use of corticosteroids along with steroid-sparing adjuvants, which allows a reduction in the total dose of corticosteroids used over the course of the treatment with a reduction in observed morbidity. The more commonly used steroid-sparing medications in the published reports include azathioprine, methotrexate, and mycophenolate mofetil. More recently, IVIg and rituximab have been used, mainly in patients with recalcitrant PV.
Overall, the mortality and morbidity from $\mathrm{PV}$ and the medications used in its treatment are considerably lower in the more recent publications than in the early reports.

The reported treatment response in patients with PV has varied significantly. Prognostic factors that have been identified include initial severity and extent of disease, with higher severity being predictive of poorer prognosis. [29]. Perhaps related to this is the fact that early initiation of treatment before the disease becomes too severe or widespread has been associated with improved prognosis [30, 31]. Once treatment is initiated, good initial response to treatment has also been found to be indicative of a better prognosis [32].

Most reports described medication courses of long duration before remission off therapy was achieved (between 5 and 10 years in the majority of patients with the range across all studies being 3 months to 27 years). However, Herbst and Bystryn described a group of 40 patients in whom 10 (25\%) patients achieved complete and long-lasting remission within 2 years of treatment; a subgroup of patients with PV, with a mild course of the disease requiring short courses of systemic medications or topical medication alone to induce remission $[5,32,33]$; and at the other extreme a subgroup that is resistant to treatment and required high doses and prolonged therapy have also been described [29, 32, 35].

The role of baseline laboratory tests, such as quantification of antibodies as predictors of disease course, has not been established. A recent study reported that a higher level of anti-Dsg1 autoantibodies $(\geq 100 \mathrm{U} / \mathrm{mL})$ at diagnosis was associated with poorer prognosis 
in univariate analyses; however, this did not remain significant after adjustment for age [36].

Periodic antibody titers measured by indirect immunofluorescence or ELISA testing have not consistently shown correlation with clinical activity of PV [37]. Most authors in the listed papers reported using clinical response alone to guide medication taper.

Reports using rituximab described remission off therapy in a shorter time frame (ranging from 2 months to 5 years) as compared to other medication combinations; this observation suggested that while the initial side effects may be significant, a shorter total duration of therapy may be possible with use of rituximab. Because rituximab is a more recent drug, first introduced in 1997, long-term side effects are not well characterized at this time.

\section{CONCLUSION}

The findings from this review emphasize the importance of early diagnosis of PV, early initiation of treatment, and use of a treatment regimen which includes a steroid-sparing adjuvant to allow a reduced total dose and duration on corticosteroids. For the majority of patients in these reports, a long-term course on medications lasting about 5-10 years was observed; however, subgroups of patients requiring shorter courses or those needing longer-term therapy were also described. In recent publications, commonly used initial regimens include corticosteroids in combination with mycophenolate or azathioprine; whereas, for patients with inadequate response to these regimens, adjuvants such as IVIG or rituximab were used $[21,38,39]$. This review also highlights the need for more controlled trials to determine optimal treatment regimens for patients with PV.

\section{ACKNOWLEDGMENTS}

No funding or sponsorship was received for this study or publication of this article. All named authors meet the International Committee of Medical Journal Editors (ICMJE) criteria for authorship of this manuscript, take responsibility for the integrity of the work as a whole, and have given final approval for the version to be published.

Disclosures. Mimansa Cholera and Nita Chainani-Wu have nothing to disclose.

Compliance with Ethics Guidelines. This article is based on previously conducted studies and does not involve any studies of human or animal subjects performed by any of the authors.

Open Access. This article is distributed under the terms of the Creative Commons Attribution-NonCommercial 4.0 International License (http://creativecommons.org/licenses/ by-nc/4.0/), which permits any noncommercial use, distribution, and reproduction in any medium, provided you give appropriate credit to the original author(s) and the source, provide a link to the Creative Commons license, and indicate if changes were made.

\section{REFERENCES}

1. Chaidemenos G, Apalla Z, Koussidou T, Papagarifallou I, Ioannides D. High dose oral prednisone vs. prednisone plus azathioprine for the treatment of oral pemphigus: a retrospective, bi-centre, comparative study. J Eur Acad Dermatol Venereol. 2011;25(2):206-10. doi:10.1111/j.14683083.2010.03753.x [Epub 2010 Jun 21].

2. Burgan SZ, Sawair FA, Napier SS. Case report: oral pemphigus vulgaris with multiple oral polyps in a young patient. Int Dent J. 2003;53(1):37-40.

3. Iamaroon A, Boonyawong $\mathrm{P}$, Klanrit $\mathrm{P}$, Prasongtunskul S, Thongprasom K. 
Characterization of oral pemphigus vulgaris in Thai patients. J Oral Sci. 2006;48(1):43-6.

4. Santoro FA, Stoopler ET, Werth VP. Pemphigus. Dent Clin North Am. 2013;57(4):597-610. doi:10. 1016/j.cden.2013.06.002 (Epub 2013 Aug 12. Review).

5. Ariyawardana A, Tilakaratne WM, Dissanayake M, et al. Oral pemphigus vulgaris in children and adolescents: a review of the literature and a case report. Int J Paediatr Dent. 2005;15(4):287-93.

6. Deyhimi P, Tavakoli P. Study of apoptosis in oral pemphigus vulgaris using immunohistochemical marker Bax and TUNEL technique. J Oral Pathol Med. 2013;42(5):409-14. doi:10.1111/jop.12022 [Epub 2012 Nov 27].

7. Endo H, Rees TD, Matsue M, Kuyama K, Nakadai M, Yamamoto H. Early detection and successful management of oral pemphigus vulgaris: a case report. J Periodontol. 2005;76(1):154-60.

8. Meurer M. Immunosuppressive therapy for autoimmune bullous diseases. Clin Dermatol. 2012;30(1):78-83. doi:10.1016/j.clindermatol. 2011.03.013.

9. Bystryn JC, Steinman NM. The adjuvant therapy of pemphigus. An update. Arch Dermatol. 1996;132(2):203-12.

10. Murrell DF, Dick S, Ahmed AR, et al. Consensus statement on definitions of disease, end points, and therapeutic response for pemphigus. J Am Acad Dermatol. 2008;58(6):1043-6. doi:10.1016/j.jaad. 2008.01.012 [Epub 2008 Mar 14].

11. Becker KL, editor. Principles and practice of endocrinology and metabolism, 3rd ed. 2001. Philadelphia: Lippincott Williams \& Wilkins

12. Lever WF, Schaumburg-Lever G. Immuno suppressants and prednisone in pemphigus vulgaris: therapeutic results obtained in 63 patients between 1961 and 1975. Arch Dermatol. 1977;113(9):1236-41.

13. Lever WF, Schaumburg-Lever G. Treatment of pemphigus vulgaris. Results obtained in 84 patients between 1961 and 1982. Arch Dermatol. 1984;120(1):44-7.

14. Lozada F, Silverman S, Cram D. Pemphigus vulgaris. A study of six cases treated with levamisole and prednisone. Oral Surg Oral Med Oral Pathol. 1982;54(2):161-5.

15. David M, Zaidenbaum M, Sandbank M. Juvenile pemphigus vulgaris: a 4- to 19-year follow-up of 4 patients. Dermatologica. 1988;177(3):165-9.
16. Lamey PJ, Rees TD, Binnie WH, Wright JM, Rankin KV, Simpson NB. Oral presentation of pemphigus vulgaris and its response to systemic steroid therapy. Oral Surg Oral Med Oral Pathol. 1992; 74(1):54-7.

17. Kaur S, Kanwar AJ. Dexamethasonecyclophosphamide pulse therapy in pemphigus. Int J Dermatol. 1990;29(5):371-4.

18. Kanwar AJ, Kaur S, Thami GP. Long-term efficacy of dexamethasone-cyclophosphamide pulse therapy in pemphigus. Dermatology. 2002;204(3):228-31.

19. Mentink LF, Mackenzie MW, Tóth GG, et al. Randomized controlled trial of adjuvant oral dexamethasone pulse therapy in pemphigus vulgaris: PEMPULS trial. Arch Dermatol. 2006;142(5):570-6 (Erratum in: Arch Dermatol. 2006 Aug;142(8):1014. Dosage error in article text).

20. Ransom JT. Mechanism of action of mycophenolate mofetil. Ther Drug Monit. 1995;17(6):681-4.

21. Powell AM, Albert S, Al Fares S, et al. An evaluation of the usefulness of mycophenolate mofetil in pemphigus. Br J Dermatol. 2003;149(1):138-45.

22. Scheinfeld NS. Intravenous immunoglobulin. Drugs Dis Proced. Medscape Reference; 2011. Web 14 Mar 2016.

23. Ruocco E, Wolf R, Ruocco V, Brunetti G, Romano F, Lo Schiavo A. Pemphigus: associations and management guidelines: facts and controversies. Clin Dermatol. 2013;31(4):382-90. doi:10.1016/j. clindermatol.2013.01.005.

24. Tsuruta D, Ishii N, Hashimoto T. Diagnosis and treatment of pemphigus. Immunotherapy. 2012;4(7):735-45.

25. Virgolini L, Marzocchi V. Anti-CD20 monoclonal antibody (rituximab) in the treatment of autoimmune diseases. Successful result in refractory pemphigus vulgaris: report of a case. Haematologica. 2003;88(7):ELT24.

26. Fleischli ME, Valek RH, Pandya AG. Pulse intravenous cyclophosphamide therapy in pemphigus. Arch Dermatol. 1999;135(1):57-61.

27. Chaffins ML, Collison D, Fivenson DP. Treatment of pemphigus and linear IgA dermatosis with nicotinamide and tetracycline: a review of 13 cases. J Am Acad Dermatol. 1993;28(6):998-1000.

28. Martin LK, Werth V, Villanueva E, Segall J, Murrell DF. Interventions for pemphigus vulgaris and pemphigus foliaceus. Cochrane Database Syst Rev. 2009;1:CD006263. doi:10.1002/14651858.CD006263. pub2. 
29. Herbst A, Bystryn JC. Patterns of remission in pemphigus vulgaris. J Am Acad Dermatol. 2000;42(3):422-7.

30. Dagistan S, Goregen M, Miloglu O, Cakur B. Oral pemphigus vulgaris: a case report with review of the literature. J Oral Sci. 2008;50(3):359-62.

31. Ben Lagha N, Poulesquen V, Roujeau JC, Alantar A, Maman L. Pemphigus vulgaris: a case-based update. J Can Dent Assoc. 2005;71(9):667-72.

32. Robinson NA, Yeo JF, Lee YS, Aw DC. Oral pemphigus vulgaris: a case report and literature update. Ann Acad Med Singapore. 2004;33(4 Suppl):63-8.

33. Camacho-Alonso F, López-Jornet P, Bermejo-Fenoll A. Pemphigus vulgaris. A presentation of 14 cases and review of the literature. Med Oral Patol Oral Cir Bucal. 2005;10(4):282-8.

34. Arin MJ, Engert A, Krieg T, Hunzelmann N. Anti-CD20 monoclonal antibody (rituximab) in the treatment of pemphigus. $\mathrm{Br} \mathrm{J}$ Dermatol. 2005;153(3):620-5.

35. Heelan K, Al-Mohammedi F, Smith MJ, et al. Durable remission of pemphigus with a fixed-dose rituximab protocol. JAMA Dermatol. 2014;150(7):703-8.

36. Baican A, Chiorean R, Leucuta DC, et al. Prediction of survival for patients with pemphigus vulgaris and pemphigus foliaceus: a retrospective cohort study. Orphanet J Rare Dis. 2015;22(10):48. doi:10.1186/ s13023-015-0263-4.

37. Cianchini G, Lupi F, Masini C, Corona R, Puddu P, De Pità $O$. Therapy with rituximab for autoimmune pemphigus: results from a single-center observational study on 42 cases with long-term follow-up. J Am Acad Dermatol. 2012;67(4):617-22. doi:10.1016/j.jaad.2011.11.007 [Epub 2012 Jan 13].

38. Chams-Davatchi C, Valikhani M, Daneshpazhooh $\mathrm{M}$, et al. Pemphigus: analysis of 1209 cases. Int J Dermatol. 2005;44(6):470-6.

39. Yazganoğlu KD, Baykal C, Küçükoğlu R. Childhood pemphigus vulgaris: five cases in 16 years. J Dermatol. 2006;33(12):846-9.

40. Ryan JG. Pemphigus. A 20-year survey of experience with 70 cases. Arch Dermatol. 1971;104(1):14-20.

41. Berger BW, Maier HS, Kantor I, Wexler DE. Pemphigus vulgaris in a $3 \frac{1}{2}-$ year old boy. Arch Dermatol. 1973;107(3):433-4. doi:10.1001/ archderm.1973.01620180083027.

42. Rosenberg FR, Sanders S, Nelson CT. Pemphigus: a 20-year review of 107 patients treated with corticosteroids. Arch Dermatol. 1976;112(7):962-70.

43. Aberer W, Wolff-Schreiner EC, Stingl G, Wolff K. Azathioprine in the treatment of pemphigus vulgaris. A long-term follow-up. J Am Acad Dermatol. 1987;16(3 Pt 1):527-33.

44. Seidenbaum M, David M, Sandbank M. The course and prognosis of pemphigus. A review of 115 patients. Int J Dermatol. 1988;27(8):580-4.

45. Laskaris G, Stoufi E. Oral pemphigus vulgaris in a 6-year-old girl. Oral Surg Oral Med Oral Pathol. 1990;69(5):609-13 (Review).

46. Werth VP. Treatment of pemphigus vulgaris with brief, high-dose intravenous glucocorticoids. Arch Dermatol. 1996;132(12):1435-9.

47. Robinson JC, Lozada-Nur F, Frieden I. Oral pemphigus vulgaris: a review of the literature and a report on the management of 12 cases. Oral Surg Oral Med Oral Pathol Oral Radiol Endod. 1997;84(4):349-55.

48. Mignogna MD, Lo Muzio L, Mignogna RE, Carbone R, Ruoppo E, Bucci E. Oral pemphigus: long term behaviour and clinical response to treatment with deflazacort in sixteen cases. J Oral Pathol Med. 2000;29(4):145-52.

49. Scully C, Paes De Almeida O, Porter SR, Gilkes JJ. Pemphigus vulgaris: the manifestations and long-term management of 55 patients with oral lesions. Br J Dermatol. 1999;140(1):84-9.

50. Ljubojević S, Lipozencić J, Brenner S, Budimcić D. Pemphigus vulgaris: a review of treatment over a 19-year period. J Eur Acad Dermatol Venereol. 2002;16(6):599-603.

51. Femiano F, Gombos F, Scully C. Pemphigus vulgaris with oral involvement: evaluation of two different systemic corticosteroid therapeutic protocols. J Eur Acad Dermatol Venereol. 2002;16(4):353-6.

52. Chaidemenos GCh, Mourellou O, Koussidou T, Tsatsou F. An alternate-day corticosteroid regimen for pemphigus vulgaris. A 13-year prospective study. J Eur Acad Dermatol Venereol. 2007;21(10):1386-91.

53. Chams-Davatchi C, Esmaili N, Daneshpazhooh M, et al. Randomized controlled open-label trial of four treatment regimens for pemphigus vulgaris. J Am Acad Dermatol. 2007;57(4):622-8 Epub 2007 Jun 21.

54. Tran KD, Wolverton JE, Soter NA. Methotrexate in the treatment of pemphigus vulgaris: experience in 23 patients. Br J Dermatol. 2013;169(4):916-21. doi:10.1111/bjd.12474. 
55. Mignogna MD, Fortuna G, Leuci S, Adamo D, Dell'Aversana Orabona G, Ruoppo E. Adjuvant triamcinolone acetonide injections in oro-pharyngeal pemphigus vulgaris. J Eur Acad Dermatol Venereol. 2010;24(10):1157-65. doi:10. 1111/j.1468-3083.2010.03610.x.

56. Mourellou O, Chaidemenos GC, Koussidou T, Kapetis E. The treatment of pemphigus vulgaris. Experience with 48 patients seen over an 11-year period. Br J Dermatol. 1995;133(1):83-7.

57. Chams-Davatchi C, Mortazavizadeh A, Daneshpazhooh $\mathrm{M}$, et al. Randomized double blind trial of prednisolone and azathioprine, vs. prednisolone and placebo, in the treatment of pemphigus vulgaris. J Eur Acad Dermatol Venereol. 2013;27(10):1285-92. doi:10.1111/j. 1468-3083.2012.04717.x [Epub 2012 Oct 15].

58. Enk AH, Knop J. Mycophenolate is effective in the treatment of pemphigus vulgaris. Arch Dermatol. 1999;135(1):54-6.

59. Grundmann-Kollmann M, Korting HC, Behrens S, et al. Mycophenolate mofetil: a new therapeutic option in the treatment of blistering autoimmune diseases. J Am Acad Dermatol. 1999;40(6 Pt 1):957-60.

60. Mimouni D, Anhalt GJ, Cummins DL, Kouba DJ, Thorne JE, Nousari HC. Treatment of pemphigus vulgaris and pemphigus foliaceus with mycophenolate mofetil. Arch Dermatol. 2003;139(6):739-42.

61. Beissert S, Werfel T, Frieling U, et al. A comparison of oral methylprednisolone plus azathioprine or mycophenolate mofetil for the treatment of pemphigus. Arch Dermatol. 2006;142(11):1447-54.

62. Strowd LC, Taylor SL, Jorizzo JL, Namazi MR. Therapeutic ladder for pemphigus vulgaris: emphasis on achieving complete remission. J Am Acad Dermatol. 2011;64(3):490-4. doi:10.1016/j. jaad.2010.02.052 [Epub 2010 Jul 14].

63. Beissert S, Mimouni D, Kanwar AJ, Solomons N, Kalia V, Anhalt GJ. Treating pemphigus vulgaris with prednisone and mycophenolate mofetil: a multicenter, randomized, placebo-controlled trial. J Invest Dermatol. 2010;130(8):2041-8. doi:10. 1038/jid.2010.91 [Epub 2010 Apr 22].

64. Bongiorno MR, Pistone G, Doukaki S, Aricò $M$. Enteric-coated mycophenolate sodium in the treatment of refractory pemphigus. Int J Dermatol. 2010;49(6):693-9. doi:10.1111/j.1365-4632.2009. 04291.x.

65. Ioannides D, Apalla Z, Lazaridou E, Rigopoulos D. Evaluation of mycophenolate mofetil as a steroid-sparing agent in pemphigus: a randomized, prospective study. J Eur Acad Dermatol Venereol. 2012;26(7):855-60. doi:10.1111/j.1468-3083.2011. 04170.x [Epub 2011 Jul 14].

66. Bystryn JC, Jiao D, Natow S. Treatment of pemphigus with intravenous immunoglobulin. J Am Acad Dermatol. 2002;47(3):358-63.

67. Amagai M, Ikeda S, Pemphigus Study Group, et al. A randomized double-blind trial of intravenous immunoglobulin for pemphigus. J Am Acad Dermatol. 2009;60(4):595-603. doi:10.1016/j.jaad. 2008.09.052.

68. Rakocević-Stojanović V, Rakocević I, Perić S, Lavrnić D. Intravenous immunoglobulin therapy in two patients with myasthenia gravis and pemphigus vulgaris. Acta Myol. 2009;28(3):101-2.

69. Lever WF, Goldberg HS. Treatment of pemphigus vulgaris with methotrexate. Arch Dermatol. 1969;100(1):70-8.

70. Jablonska S, Chorzelski T, Blaszczyk M. Immunosuppressants in the treatment of pemphigus. Br J Dermatol. 1970;83(2):315-23 (Review).

71. Piamphongsant T, Sivayathorn A. Pemphigus: combined treatment with methotrexate and prednisone. J Med Assoc Thai. 1975;58(3):171-6.

72. Lever WF, Schaumburg-Lever G. Immunosuppressants and prednisone in pemphigus vulgaris: therapeutic results obtained in 63 patients between 1961 and $1975 . \quad$ Arch Dermatol. 1977;113(9):1236-41.

73. Lever WF. Methotrexate and prednisone in pemphigus vulgaris. Therapeutic results obtained in 36 patients between 1961 and 1970. Arch Dermatol. 1972;106(4):491-7.

74. Mashkilleyson N, Mashkilleyson AL. Mucous membrane manifestations of pemphigus vulgaris. A 25-year survey of 185 patients treated with corticosteroids or with combination of corticosteroids with methotrexate or heparin. Acta Derm Venereol. 1988;68(5):413-21.

75. Smith TJ, Bystryn JC. Methotrexate as an adjuvant treatment for pemphigus vulgaris. Arch Dermatol. 1999;135(10):1275-6.

76. Baum S, Greenberger S, Samuelov L, et al. Methotrexate is an effective and safe adjuvant therapy for pemphigus vulgaris. Eur J Dermatol. 2012;22(1):83-7. doi:10.1684/ejd.2011.1611.

77. Salopek TG, Logsetty S, Tredget EE. Anti-CD20 chimeric monoclonal antibody (rituximab) for the 
treatment of recalcitrant, life-threatening pemphigus vulgaris with implications in the pathogenesis of the disorder. J Am Acad Dermatol. 2002;47(5):785-8.

78. Cooper HL, Healy E, Theaker JM, Friedmann PS. Treatment of resistant pemphigus vulgaris with an anti-CD20 monoclonal antibody (rituximab). Clin Exp Dermatol. 2003;28(4):366-8.

79. España A, Fernández-Galar M, Lloret $P$, Sánchez-Ibarrola A, Panizo C. Long-term complete remission of severe pemphigus vulgaris with monoclonal anti-CD20 antibody therapy and immunophenotype correlations. J Am Acad Dermatol. 2004;50(6):974-6.

80. Morrison LH. Therapy of refractory pemphigus vulgaris with monoclonal anti-CD20 antibody (rituximab). J Am Acad Dermatol. 2004;51(5):817-9.

81. Wenzel J, Bauer R, Bieber T, Tüting T. Successful rituximab treatment of severe pemphigus vulgaris resistant to multiple immunosuppressants. Acta Derm Venereol. 2005;85(2):185-6.

82. Dupuy A, Viguier M, Bédane C, et al. Treatment of refractory pemphigus vulgaris with rituximab (anti-CD20 monoclonal antibody). Arch Dermatol. 2004;140(1):91-6.

83. Kong HH, Prose NS, Ware RE, Hall RP 3rd. Successful treatment of refractory childhood pemphgus vulgaris with anti-CD20 monoclonal antibody (rituximab). Pediatr Dermatol. 2005;22(5):461-4.

84. Schmidt E, Herzog S, Bröcker EB, Zillikens D, Goebeler M. Long-standing remission of recalcitrant juvenile pemphigus vulgaris after adjuvant therapy with rituximab. Br J Dermatol. 2005;153(2):449-51.

85. Schmidt E, Seitz CS, Benoit S, Bröcker EB, Goebeler M. Rituximab in autoimmune bullous diseases: mixed responses and adverse effects. $\mathrm{Br} J$ Dermatol. 2007;156(2):352-6.

86. Ahmed AR, Spigelman Z, Cavacini LA, Posner MR. Treatment of pemphigus vulgaris with rituximab and intravenous immune globulin. N Engl J Med. 2006;355(17):1772-9.

87. Goh MS, McCormack C, Dinh HV, Welsh B, Foley P, Prince HM. Rituximab in the adjuvant treatment of pemphigus vulgaris: a prospective open-label pilot study in five patients. $\mathrm{Br} \mathrm{J}$ Dermatol. 2007;156(5):990-6 [Epub 2007 Mar 13].

88. Marzano AV, Fanoni D, Venegoni L, Berti E, Caputo R. Treatment of refractory pemphigus with the
anti-CD20 monoclonal antibody (rituximab). Dermatology. 2007;214(4):310-8.

89. Antonucci A, Negosanti M, Tabanelli M, Varotti C. Treatment of refractory pemphigus vulgaris with anti-CD20 monoclonal antibody (rituximab): five cases. J Dermatol Treat. 2007;18(3):178-83.

90. Cianchini G, Corona R, Frezzolini A, Ruffelli M, Didona B, Puddu P. Treatment of severe pemphigus with rituximab: report of 12 cases and a review of the literature. Arch Dermatol. 2007;143(8):1033-8 (Review).

91. Joly P, Mouquet H, Roujeau JC, et al. A single cycle of rituximab for the treatment of severe pemphigus. N Engl J Med. 2007;357(6):545-52.

92. Shimanovich I, Nitschke M, Rose C, Grabbe J, Zillikens D. Treatment of severe pemphigus with protein A immunoadsorption, rituximab and intravenous immunoglobulins. $\mathrm{Br} \mathrm{J}$ Dermatol. 2008;158(2):382-8 [Epub 2007 Dec 6].

93. Eming R, Nagel A, Wolff-Franke S, Podstawa E, Debus D, Hertl M. Rituximab exerts a dual effect in pemphigus vulgaris. J Invest Dermatol. 2008;128(12):2850-8. doi:10.1038/jid.2008.172 [Epub 2008 Jun 19].

94. Faurschou A, Gniadecki R. Two courses of rituximab (anti-CD20 monoclonal antibody) for recalcitrant pemphigus vulgaris. Int J Dermatol. 2008;47(3):292-4. doi:10.1111/j.1365-4632.2008.03423.x.

95. Pfütze M, Eming R, Kneisel A, Kuhlmann U, Hoyer J, Hertl M. Clinical and immunological follow-up of pemphigus patients on adjuvant treatment with immunoadsorption or rituximab. Dermatology. 2009;218(3):237-45. doi:10.1159/000187431 Epub 2008 Dec 16.

96. Fuertes I, Guilabert A, Mascaró JM Jr, Iranzo P. Rituximab in childhood pemphigus vulgaris: a long-term follow-up case and review of the literature. Dermatology. 2010;221(1):13-6. doi:10. 1159/000287254 (Epub 2010 Apr 9. Review).

97. Kasperkiewicz M, Shimanovich I, Meier M, et al. Treatment of severe pemphigus with a combination of immunoadsorption, rituximab, pulsed dexamethasone and azathioprine/mycophenolate mofetil: a pilot study of 23 patients. $\mathrm{Br} \mathrm{J}$ Dermatol. 2012;166(1):154-60. doi:10.1111/j.13652133.2011.10585.x [Epub 2011 Oct 17].

98. Craythorne E, du Viver A, Mufti GJ, Warnakulasuriya S. Rituximab for the treatment of corticosteroid-refractory pemphigus vulgaris with oral and skin manifestations. J Oral Pathol Med. 2011;40(8):616-20. doi:10.1111/j.1600-0714.2011. 01017.x [Epub 2011 Mar 9]. 
99. Kasperkiewicz M, Shimanovich I, Ludwig RJ, Rose C, Zillikens D, Schmidt E. Rituximab for treatment-refractory pemphigus and pemphigoid: a case series of 17 patients. J Am Acad Dermatol. 2011;65(3):552-8. doi:10.1016/j. jaad.2010.07.032.

100. Kim JH, Kim YH, Kim MR, Kim SC. Clinical efficacy of different doses of rituximab in the treatment of pemphigus: a retrospective study of 27 patients. $\mathrm{Br} \mathrm{J}$ Dermatol. 2011;165(3):646-51. doi:10.1111/j.13652133.2011.10411.x.

101. Reguiai Z, Tabary $T$, Maizières $M$, Bernard $P$. Rituximab treatment of severe pemphigus: long-term results including immunologic follow-up. J Am Acad Dermatol. 2012;67(4):623-9. doi:10.1016/j.jaad.2011.12.019 [Epub 2012 Jan 20].

102. Horváth B, Huizinga J, Pas $\mathrm{HH}$, Mulder AB, Jonkman MF. Low-dose rituximab is effective in pemphigus. Br J Dermatol. 2012;166(2):405-12. doi:10.1111/j.1365-2133.2011.10663.x [Epub 2012 Jan 9].

103. Feldman RJ, Christen WG, Ahmed AR. Comparison of immunological parameters in patients with pemphigus vulgaris following rituximab and IVIG therapy. Br J Dermatol. 2012;166(3):511-7. doi:10. 1111/j.1365-2133.2011.10658.x [Epub 2012 Jan 19].

104. Leshem YA, Hodak E, David M, Anhalt GJ, Mimouni D. Successful treatment of pemphigus with biweekly 1-g infusions of rituximab: a retrospective study of 47 patients. J Am Acad Dermatol. 2013;68(3):404-11. doi:10.1016/j.jaad. 2012.08.010 [Epub 2012 Oct 6].

105. Lunardon L, Tsai KJ, Propert KJ, et al. Adjuvant rituximab therapy of pemphigus: a single-center experience with 31 patients. Arch Dermatol. 2012;148(9):1031-6. doi:10.1001/archdermatol. 2012.1522 .

106. Kasperkiewicz M, Eming R, Behzad M, Hunzelmann $\mathrm{N}$, et al. Efficacy and safety of rituximab in pemphigus: experience of the German Registry of
Autoimmune Diseases. J Dtsch Dermatol Ges. 2012;10(10):727-32. doi:10.1111/j.1610-0387. 2012.07931.x [Epub 2012 May 11].

107. Balighi K, Daneshpazhooh M, Khezri S, Mahdavi-nia M, Hajiseyed-javadi M, Chams-Davatchi C. Adjuvant rituximab in the treatment of pemphigus vulgaris: a phase II clinical trial. Int J Dermatol. 2013;52(7):862-7. doi:10.1111/j.1365-4632.2012.5847.x (Erratum in: Int J Dermatol. 2013 Oct;52(10):1292. Kamran, Balighi [corrected to Balighi, Kamran]; Maryam, Daneshpazhooh [corrected to Daneshpazhooh, Maryam]; Somayeh, Khezri [corrected to Khezri, Somayeh]; Mostafa, Mahdavi-nia [corrected to Mahdavi-nia, Mostafa]; Mahsa, Hajiseyed-javadi [corrected to Hajiseyed-javadi, Mahsa]; Cheyda, Chams-Davatchi [corrected to Chams-Davatchi, Cheyda]).

108. Kanwar AJ, Tsuruta D, Vinay K, et al. Efficacy and safety of rituximab treatment in Indian pemphigus patients. J Eur Acad Dermatol Venereol. 2013;27(1):e17-23. doi:10.1111/j.1468-3083.2011. 04391.x [Epub 2011 Dec 17].

109. Kolesnik M, Becker E, Reinhold D, et al. Treatment of severe autoimmune blistering skin diseases with combination of protein A immunoadsorption and rituximab: a protocol without initial high dose or pulse steroid medication. J Eur Acad Dermatol Venereol. 2014;28(6):771-80. doi:10.1111/jdv. 12175 [Epub 2013 May 8].

110. Kanwar AJ, Vinay K, Sawatkar GU, et al. Clinical and immunological outcomes of high- and low-dose rituximab treatments in patients with pemphigus: a randomized, comparative, observer-blinded study. Br J Dermatol. 2014;170(6):1341-9. doi:10.1111/ bjd.12972.

111. Ojaimi S, O'Connor K, Lin MW, Schifter M, Fulcher DA. Treatment outcomes in cohort of patients with mucosal-predominant pemphigus vulgaris. Intern Med J. 2015;45(3):284-92. doi:10.1111/imj.12674. 